\title{
Limits of JT gravity
}

\author{
Daniel Grumiller, ${ }^{a, 1}$ Jelle Hartong, ${ }^{b, 2}$ Stefan Prohazka ${ }^{b, 3}$ and Jakob Salzer ${ }^{c, 4}$ \\ ${ }^{a}$ Institute for Theoretical Physics, TU Wien, \\ Wiedner Hauptstrasse 8-10/136, A-1040 Vienna, Austria \\ ${ }^{b}$ School of Mathematics and Maxwell Institute for Mathematical Sciences, University of Edinburgh, \\ Peter Guthrie Tait Road, Edinburgh EH9 3FD, U.K. \\ ${ }^{c}$ Center for the Fundamental Laws of Nature, Harvard University, \\ Cambridge, MA 02138, U.S.A. \\ E-mail: grumil@hep.itp.tuwien.ac.at, jelle.hartong@ed.ac.uk, \\ stefan.prohazka@ed.ac.uk, jsalzer@fas.harvard.edu
}

ABSTRACT: We construct various limits of JT gravity, including Newton-Cartan and Carrollian versions of dilaton gravity in two dimensions as well as a theory on the threedimensional light cone. In the $\mathrm{BF}$ formulation our boundary conditions relate boundary connection with boundary scalar, yielding as boundary action the particle action on a group manifold or some Hamiltonian reduction thereof. After recovering in our formulation the Schwarzian for JT, we show that AdS-Carroll gravity yields a twisted warped boundary action. We comment on numerous applications and generalizations.

Keywords: 2D Gravity, Classical Theories of Gravity, Space-Time Symmetries

ARXIV EPRINT: 2011.13870

\footnotetext{
${ }^{1}$ ORCID: 0000-0001-7980-5394

${ }^{2}$ ORCID: 0000-0003-0498-0029

${ }^{3}$ ORCID: 0000-0002-3925-3983

${ }^{4}$ ORCID: 0000-0002-9560-344X
} 


\section{Contents}

1 Introduction 2

2 2d gauge theories of gravity 3

2.1 BF theories 3

2.2 Geometric interpretation 4

3 Limits of JT gravity 5

3.1 AdS and dS BF theory 5

$\begin{array}{lll}3.2 & \mathrm{BF} \text { on the light cone } & 6\end{array}$

$\begin{array}{lll}3.3 & \text { Kinematical limits of BF theories } & 7\end{array}$

3.4 Newton-Cartan dilaton gravity and Carroll dilaton gravity 11

4 Metric BF theories and their limits $\quad 14$

$\begin{array}{lll}4.1 & \text { Metrics and limits } & 14\end{array}$

$\begin{array}{lll}4.2 & \text { Flat space dilaton gravity } & 14\end{array}$

$\begin{array}{lll}4.3 \text { Summary } & 15\end{array}$

5 Boundary actions of kinematical BF theories $\quad 17$

$\begin{array}{lll}5.1 & \text { Particle on group manifold } & 17\end{array}$

$\begin{array}{lll}5.2 \text { Hamiltonian reduction of boundary action } & 19\end{array}$

6 Schwarzian-like theories $\quad 20$

6.1 Euclidean $\mathrm{AdS}_{2} /$ Hyperbolic plane 20

$\begin{array}{lll}6.2 \text { AdS-Carroll }_{2} & 23\end{array}$

$\begin{array}{lll}6.3 & \text { Other kinematical algebras } & 25\end{array}$

7 Applications and generalizations $\quad 26$

$\begin{array}{lll}7.1 & \text { Selected applications } & 26\end{array}$

$\begin{array}{lll}7.2 & \text { Selected generalizations } & 29\end{array}$

A Metric Lie algebras of low dimension $\quad 30$

B Lifshitz, Schrödinger and 1/c expanded BF theories 31

C Coadjoint theories and their limits $\quad 32$

$\begin{array}{ll}\text { D Matrix representations } & 33\end{array}$ 


\section{Introduction}

Jackiw-Teitelboim (JT) gravity [1-4] features prominently in classical and quantum gravity as a convenient toy model to elucidate conceptual problems while keeping the technical ones at a bare minimum. Examples include implementing 't Hooft's brick wall proposal [5], Cardyology attempts [6], noncommutative geometry [7], holographic renormalization and thermodynamics [8], the attractor mechanism [9], constant dilaton holography [10, 11], the JT/SYK correspondence [12] (for reviews see [13-15]), relations to random matrix models $[16,17], T \bar{T}$-deformations $[18,19]$, traversable wormholes [20], holographic complexity [21], constructions of the Hartle-Hawking wavefunction [22, 23] and implementations of the island proposal to resolve the black information loss problem [24-29]. See [30] for a review on further aspects of two-dimensional (2d) dilaton gravity, including numerous generalizations of JT gravity, like the Callan-Giddings-Harvey-Strominger (CGHS) model [31]. None of these generalizations so far gave up the assumption of (pseudo-)Riemannian metrics (or a corresponding Cartan formulation).

For applications or toy models of non-relativistic holography it is of interest to consider singular limits of JT gravity to, say, Carrollian or Galilean spacetimes. The main purpose of our work is to show how this is done and to discuss some aspects of these new models, including boundary actions and boundary conditions. Among other applications our construction allows to address questions such as "Is there a Newton-Cartan version of 2d dilaton gravity?" or "What is the Schwarzian analogue for the AdS-Carroll limit of JT gravity?".

The urge to look for theories beyond the Riemann-Cartan setup is partly motivated by the relation of Carrollian symmetries to null surfaces, like horizons or null infinity in flat space, and partly by applications of non-relativistic theories in condensed matter physics. Eventually, some of our models may serve as gravity duals for examples of nonor ultra-relativistic holography in the spirit of the JT/SYK correspondence, and many of the questions addressed and issues raised in this context could potentially be transposed to models introduced in our work.

In several ways this work mirrors investigations of Chern-Simons theories in $2+1$ dimensions based on Lie algebras beyond the semi-simple case, started in [32, 33] for Poincaré and (A)dS and extended to Galilei [34, 35] and beyond [36-39] and to higher spins [40].

This paper is organized as follows. In section 2 we review general aspects of the formulation of $2 \mathrm{~d}$ gravity as a gauge theory of BF-type. In section 3 we take singular limits of JT gravity, among other things to Galilean and Carrollian theories that we generalize to Newton-Cartan and Carroll dilaton gravity; we also show that there is a light cone theory that does not require any limit. In section 4 we focus on the subclass of metric BF theories and their limits. In section 5 we discuss boundary actions and how to perform a Hamiltonian reduction from the action for a particle moving on a group manifold to a Schwarzian-like action by imposing certain constraints. In section 6 we discuss two examples for such boundary actions, first for JT and then for AdS-Carroll 2 gravity. In section 7 we conclude with a discussion of possible applications and generalizations. 
Notation. When applicable, upper (lower) signs in equations refer to the Euclidean (Lorentzian) case.

Note added. Shortly after our work [41] appeared on the arXiv. Where applicable, our results agree with each other.

\section{$2 \quad 2 d$ gauge theories of gravity}

JT gravity in its first order formulation $[42,43]$ is a specific BF theory based on the Lie algebra $\mathfrak{s o}(2,1)$, which features an invariant metric. Some of its limits may lead to Lie algebras without metric. Since these subtleties will be relevant for the remaining work, we set the stage by providing a rather detailed reminder of BF theories.

We follow [44] where further details are provided (see also the review [45]; especially relevant is section 6 on Schwarz type topological gauge theories).

\subsection{BF theories}

$B F$ theory is defined by the bulk action

$$
\begin{aligned}
I^{\mathrm{BF}}\left[\mathcal{X}^{*}, A\right] & =\frac{k}{2 \pi} \int_{\mathcal{M}^{2}} \mathscr{L}^{\mathrm{BF}}\left[\mathcal{X}^{*}, A\right] \\
\mathscr{L}^{\mathrm{BF}}\left[\mathcal{X}^{*}, A\right] & =\mathcal{X}^{*} F=X_{K}\left(d A^{K}+\frac{1}{2} c_{I J}{ }^{K} A^{I} \wedge A^{J}\right)
\end{aligned}
$$

where $k$ is a dimensionless coupling constant, $\mathcal{X}^{*}=X_{I} \mathrm{E}^{I}$ is a scalar transforming in the coadjoint representation and the Lie algebra valued one-form $A=A_{\mu}^{I} \mathrm{e}_{I} d x^{\mu}$ is a gauge field with curvature two-form $F \equiv d A+\frac{1}{2}[A, A]$. The structure constants $c_{I J}{ }^{K}$ of a Lie algebra $\mathfrak{g}$ are defined by $\left[\mathrm{e}_{I}, \mathrm{e}_{J}\right]=c_{I J}{ }^{K} \mathrm{e}_{K}$ (with the dual $\mathfrak{g}^{*}$ with basis $\mathrm{E}^{I}$ given by $\left.\mathrm{E}^{I}\left(\mathrm{e}_{J}\right)=\delta_{J}^{I}\right)$. For $X, Y \in \mathfrak{g}$ and $\mathcal{Y} \in \mathfrak{g}^{*}$ the adjoint and coadjoint actions are given by $\operatorname{ad}_{X} Y=[X, Y]$ and $\operatorname{ad}_{X}^{*} \mathcal{Y}(\cdot)=-\mathcal{Y}\left(\operatorname{ad}_{X} \cdot\right)$, respectively. Equivalently, in a basis this reads $\operatorname{ad}_{\mathrm{e}_{I}} \mathrm{e}_{J}=\left[\mathrm{e}_{I}, \mathrm{e}_{J}\right]=c_{I J}{ }^{K} \mathrm{e}_{K}$ and $\operatorname{ad}_{e_{I}}^{*} \mathrm{E}^{J}=-c_{I K}{ }^{J} \mathrm{E}^{K}$.

The definition of $\mathrm{BF}$ theory does not require a trace or invariant metric. This is different from gauge theories of, e.g., Chern-Simons or Yang-Mills type that are based on Lie algebras with an invariant metric. The gauge transformations are given by $\delta_{\lambda} \mathcal{X}^{*}=\operatorname{ad}_{\lambda}^{*} \mathcal{X}^{*}$ and $\delta_{\lambda} A=-(d \lambda+[A, \lambda])$ (and hence $\delta_{\lambda} F=\operatorname{ad}_{\lambda} F=[\lambda, F]$ ), or explicitly,

$$
\delta_{\lambda} X_{I}=c_{I J}{ }^{K} \lambda^{J} X_{K} \quad \delta_{\lambda} A^{I}=-d \lambda^{I}-c_{J K}{ }^{I} A^{J} \lambda^{K}
$$

and leave invariant the action (2.1). Varying it leads to the equations of motion

$$
F^{I}=d A^{I}+\frac{1}{2} c_{J K}^{I} A^{J} \wedge A^{K}=0 \quad d X_{I}+c_{I J}{ }^{K} A^{J} X_{K}=0 .
$$

An interesting subclass of BF theories is obtained when the gauge algebra is given by a metric or (regular) quadratic Lie algebra. This means that the Lie algebra admits an invariant metric $\langle\cdot, \cdot\rangle: \mathfrak{g} \times \mathfrak{g} \rightarrow \mathbb{R}$ that is a non-degenerate, symmetric, ad-invariant bilinear form (in the following we will often omit the comma; by ad-invariance we mean 
$\langle[z, x], y\rangle+\langle x,[z, y]\rangle=0$ for all Lie algebra elements $x, y, z \in \mathfrak{g})$. Since it is non-degenerate, we can use this metric to identify elements of the dual $\mathfrak{g}^{*}$ with elements of the Lie algebra $\mathfrak{g}$ via $\langle\mathcal{X}, \cdot\rangle=\mathcal{X}^{*}(\cdot)$, or more explicitly $X^{I}=g^{I J} X_{J}$ (where $g_{I J}=\left\langle\mathrm{e}_{I}, \mathrm{e}_{J}\right\rangle$ and $g^{I J} g_{J K}=\delta_{K}^{I}$ ).

The Lagrangian for metric BF theory is given by

$$
\mathscr{L}^{\mathrm{mBF}}[\mathcal{X}, A]=\langle\mathcal{X}, F\rangle=g_{L K} X^{L}\left(d A^{K}+\frac{1}{2} c_{I J}{ }^{K} A^{I} \wedge A^{J}\right)
$$

with equations of motion

$$
F=0 \quad d \mathcal{X}+[A, \mathcal{X}]=0 .
$$

As the coadjoint and adjoint representations are isomorphic we rewrite the transformation $\delta_{\lambda} \mathcal{X}=\operatorname{ad}_{\lambda} \mathcal{X}=[\lambda, \mathcal{X}]$. The standard example of metric BF theories are given by simple Lie algebras where one can use the matrix trace to write $\mathscr{L}^{\mathrm{mBF}}=\operatorname{tr}(\mathcal{X} F)$. There indeed exist Lie algebras beyond the semisimple ones that admit an invariant metric, e.g., one notable example is the $2+1$ dimensional Poincaré algebra. There exists a structure theorem that helps to understand them [46] (see also [47]) which has also been used to find generalizations for the Galilei and Carrollian cases which we will encounter below [39]. We provide an overview of all Lie algebras of low dimension that admit an invariant metric in appendix A.

\subsection{Geometric interpretation}

Up until now the BF theory (2.1), or metric BF theory (2.4), defines a (topological) gauge theory based on a gauge algebra $\mathfrak{g}$ without any geometrical meaning. In this subsection we clarify how the fields appearing in these actions acquire a geometric interpretation upon introducing additional structure in the form of a Klein pair. To this end it is convenient to introduce the notion of kinematical spacetime.

As is well-known, (A)dS and Minkowski space, and quotients thereof, are the only Lorentzian manifolds that are both homogeneous and isotropic. In $n$ spacetime dimensions this implies the existence of $n(n+1) / 2$ Killing vectors. From this follows that one can describe these spacetimes, without introducing a metric, as the homogeneous spaces $G / \mathrm{SO}(n-1,1)$ with $G=\mathrm{SO}(n-1,2)$ for AdS, $G=\mathrm{SO}(n, 1)$ for dS, and $G=\operatorname{ISO}(n-1,1)$ for Minkowski, respectively. In addition to being homogeneous, all these spacetimes are also isotropic. By this we mean that the symmetry group contains $\mathrm{SO}(n-1)$ as a subgroup and splits into representations thereof.

More generally, one can ask the question which other homogeneous and isotropic spaces, i.e., kinematical spacetimes, of dimensions $n$ exist. This question, studied first in [48] and answered exhaustively in [49], boils down to a classification of so-called Klein pairs $(\mathfrak{g}, \mathfrak{h})$ with $\mathfrak{h}$ an $n(n-1) / 2$ dimensional subalgebra of the $n(n+1) / 2$ dimensional Lie algebra $\mathfrak{g}$ that together determine the respective kinematical spacetimes. Note that most of these spacetimes do not exhibit a metric of Lorentzian or Euclidean signature but can be classified according to the existence of an ultra-relativistic Carrollian or a non-relativistic Galilean structure. The former consists of a degenerate metric whose kernel is spanned by a single vector field. A Galilean structure, on the other hand, is defined by a degenerate 
co-metric whose kernel is spanned by a nowhere vanishing one-form. One can furthermore define, in most cases, a distinguished connection on these spacetimes and classify these spacetimes according to the curvature of this connection. ${ }^{1}$ For more details we refer the reader to [49]. We emphasize again that the choice of subalgebra $\mathfrak{h}$ is important as spacetimes with isomorphic symmetry algebra $\mathfrak{g}$ can have vastly different geometric properties depending on the choice of $\mathfrak{h}$. We will encounter examples illustrating this fact throughout this work.

Returning to the problem at hand, we denote the three generators of $\mathfrak{g}$ in $1+1$ dimensions as $\mathfrak{g}=\{\mathrm{H}, \mathrm{P}, \mathrm{B}\}$ and use the convention that $\mathfrak{h}$ is always spanned by the generator denoted by B. Expanding the Lie algebra-valued one-form of a BF theory based on this algebra as

$$
A=\tau \mathrm{H}+e \mathrm{P}+\omega \mathrm{B}
$$

we can now interpret the field associated to $\mathrm{H}(\mathrm{P})$ as temporal (spatial) zweibein component. The field $\omega$ is the gauge field of the internal symmetry transformation, i.e., the dualized spin-connection associated to local Lorentz transformations in the relativistic case. The Klein pair thus provides a map from the a priori abstract gauge field to geometric data.

\section{Limits of JT gravity}

To set the stage we briefly review the well-known (A)dS JT gravity case in BF formulation. Next we define a novel BF theory on the light cone, which is based on the same simple Lie algebra, but the underlying spacetimes differ due to different geometric interpretations of the gauge connection components. Then we discuss and provide the kinematical limits of (A)dS JT gravity.

\subsection{AdS and dS BF theory}

The AdS $(\hat{\Lambda}<0)$ and $\mathrm{dS}(\hat{\Lambda}>0)$ BF theories can be written in a covariant fashion where we use $\mathrm{P}_{a}=\left(\mathrm{P}_{0}, \mathrm{P}_{1}\right)=(\mathrm{H}, \mathrm{P})$. They are based on the Lie algebra

$$
\left[\mathrm{B}, \mathrm{P}_{a}\right]=-\epsilon_{a}{ }^{b} \mathrm{P}_{b} \quad\left[\mathrm{P}_{a}, \mathrm{P}_{b}\right]=-\hat{\Lambda} \epsilon_{a b} \mathrm{~B}
$$

where $\epsilon_{01}=1$ and we raise and lower with $\eta_{a b}=\operatorname{diag}(1,-1)$ in the Lorentzian and $\eta_{a b}=\delta_{a b}=\operatorname{diag}(1,1)$ in the Euclidean case. The most general invariant metric is

$$
\langle\mathrm{B}, \mathrm{B}\rangle=\mu \quad\left\langle\mathrm{P}_{a}, \mathrm{P}_{b}\right\rangle=\mu \hat{\Lambda} \eta_{a b}
$$

where $\mu \neq 0$ is an overall proportionality factor.

The Lie algebras for positive and negative $\hat{\Lambda}$ are isomorphic. It is the choice which generator is part of the spin-connection and which is part of the vielbein that provides the distinction between these two cases. As discussed in the previous section, assuming that $B$ spans the subalgebra $\mathfrak{h}$, the Klein pair dictates the form

$$
A=\omega \mathrm{B}+e^{a} \mathrm{P}_{a} \quad \mathcal{X}=X \mathrm{~B}+X^{a} \mathrm{P}_{a}
$$

\footnotetext{
${ }^{1}$ For non-homogeneous Carrollian or Galilean spacetimes there does not exists a preferred connection, like the Levi-Civita connection in the case of Lorentzian geometries [50-52].
} 


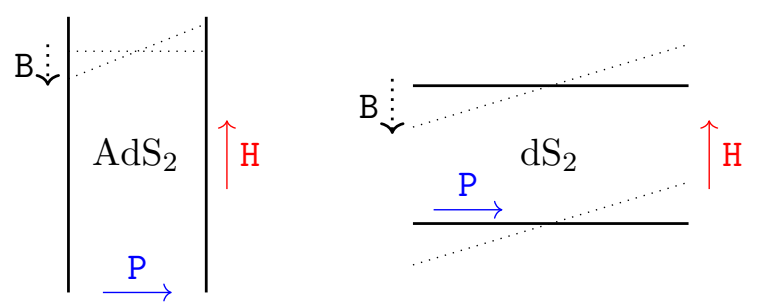

Figure 1. Penrose diagrams for $\mathrm{AdS}_{2}$ (left) and $\mathrm{dS}_{2}$ (right).

of the gauge field and the coadjoint scalar. Together with the metric (3.2) this allows us to write the Lagrangian for (A)dS-JT gravity as

$$
\mathcal{L}_{\mathrm{JT}}=X\left(d \omega-\frac{\hat{\Lambda}}{2} e^{a} \wedge e^{b} \epsilon_{a b}\right)+X^{a}\left(d e_{a}+\epsilon_{a}{ }^{b} \omega \wedge e_{b}\right)
$$

where we rescaled $\hat{\Lambda} X^{a} \rightarrow X^{a}$ for convenience. The fields $X^{a}$ enforce the $2 \mathrm{~d}$ torsion constraint for the zweibein $e^{a}$. Upon solving this for the spin connection $\omega$ and plugging it into the action, one is left with the well-known second-order action for JT gravity with $X$ being the dilaton field.

As a reminder the Penrose diagrams of $(\mathrm{A}) \mathrm{dS}_{2}$ are depicted in figure 1 , where the transformations generated by translations $\mathrm{H}, \mathrm{P}$ and boosts $\mathrm{B}$ are indicated.

For later purposes we discuss now briefly how to arrive at arbitrary dilaton gravity models starting from JT. The JT Lagrangian (3.4) has as most general Lorentz invariant generalization that preserves the Palatini condition of vanishing on-shell torsion the Lagrangian

$$
\mathcal{L}_{\text {dil }}=X\left(d \omega+V(X) e^{a} \wedge e^{b} \epsilon_{a b}\right)+X^{a}\left(d e_{a}+\epsilon_{a}^{b} \omega \wedge e_{b}\right) .
$$

that depends on an arbitrary function of the dilaton field, $V(X)$. (Dropping the Palatini condition further generalizes $V(X) \rightarrow V\left(X, X^{a} X_{a}\right)$.) Thus, JT naturally generalizes to generic dilaton gravity models. The same is true for its various limits studied below.

\subsection{BF on the light cone}

In addition to the well-known homogeneous spaces and their BF theories mentioned in the previous subsection there exists another homogeneous space based on the symmetry algebra $\mathfrak{s l}(2, \mathbb{R})$ which is the light cone of three dimensional Minkowski space seen as $2 \mathrm{~d}$ manifold. The homogeneous space of the light cone is based on the following algebra, see, e.g., [49]

$$
[\mathrm{B}, \mathrm{H}]=-\mathrm{B} \quad[\mathrm{B}, \mathrm{P}]=\mathrm{H} \quad[\mathrm{H}, \mathrm{P}]=-\mathrm{P}
$$

and invariant metric

$$
\langle\mathrm{B}, \mathrm{P}\rangle=\mu \quad\langle\mathrm{H}, \mathrm{H}\rangle=\mu .
$$

The Lie algebra is, for any dimension, isomorphic to the one of de Sitter spacetime [49] (in $1+1$ dimensions also to the one of anti-de Sitter). However due to the different choice of 


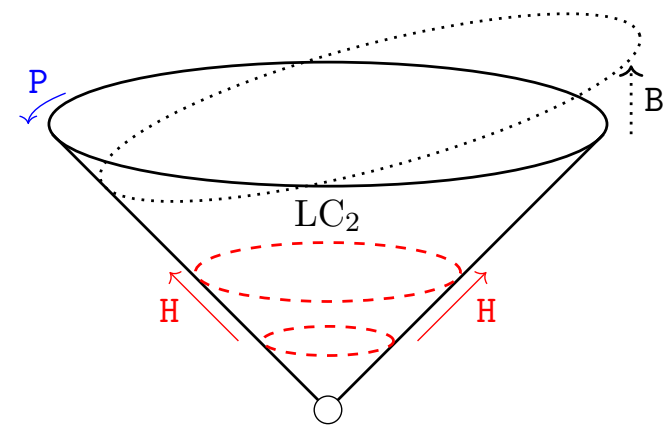

Figure 2. Two-dimensional future light cone of three-dimensional Minkowski space with vertex removed. Topologically, this is a strip like $(\mathrm{A}) \mathrm{dS}_{2}$.

subalgebra $\mathfrak{h}$, the corresponding homogeneous spaces differ. In particular, the light cone is a Carrollian spacetime, i.e., one can define an invariant Carrollian structure on this spacetime; see [49] for the explicit construction.

In a sense the light cone is in between $\mathrm{AdS}_{2}$ (which has timelike asymptotic boundaries) and $\mathrm{dS}_{2}$ (which has spacelike asymptotic boundaries). Instead of a Penrose diagram we just depict the light cone itself in figure 2, together with the geometric interpretation of the three generators.

The light cone theory has the Lagrangian

$$
\mathcal{L}_{\mathrm{LC}}=\langle\mathcal{X}, F\rangle=X^{\mathrm{H}}(d \tau+\omega \wedge e)+X^{\mathrm{P}}(d e-\tau \wedge e)+X(d \omega-\omega \wedge \tau)
$$

where we used

$$
\mathcal{X}=X^{\mathrm{P}} \mathrm{B}+X^{\mathrm{H}} \mathrm{H}+X \mathrm{P} \quad A=\omega \mathrm{B}+\tau \mathrm{H}+e \mathrm{P} .
$$

This Lagrangian is equivalent to the JT Lagrangian, but the interpretation is different since 'boosts' generated by B act differently in these two theories (see figures 1 and 2).

\subsection{Kinematical limits of BF theories}

As discussed in section 2.1 BF theories allow for a gauge invariant action, irrespective of the existence of an invariant metric on the Lie algebra. This means we can take limits without compromising the well-definedness of the action and theory. Before we show this we introduce an additional generator $M$ into our theory such that the nonzero commutators are given by

$$
[\mathrm{B}, \mathrm{H}]=\mp \hat{C}^{2} \mathrm{P} \quad[\mathrm{B}, \mathrm{P}]=\hat{c}^{2} \mathrm{H}+\alpha \mathrm{M} \quad[\mathrm{H}, \mathrm{P}]=-\hat{\Lambda} \mathrm{B} .
$$

The upper (lower) sign specifies that the theory is Euclidean (Lorentzian) in case this distinction is applicable and $\hat{\Lambda}$ is the cosmological constant which is (negative) positive for $(\mathrm{A}) \mathrm{dS}$ spacetimes. Each of the flat $(\hat{\Lambda} \rightarrow 0)$, Galilean $\left(\hat{c}=\frac{1}{c} \rightarrow 0\right)^{2}$ or Carrollian $(\hat{C} \rightarrow 0)$ limits leads again to a well-defined Lie algebra; for a visualization of these limits

\footnotetext{
${ }^{2}$ The inverse speed of light $\hat{c}$ is introduced so that all contractions involve parameters tending to zero.
} 


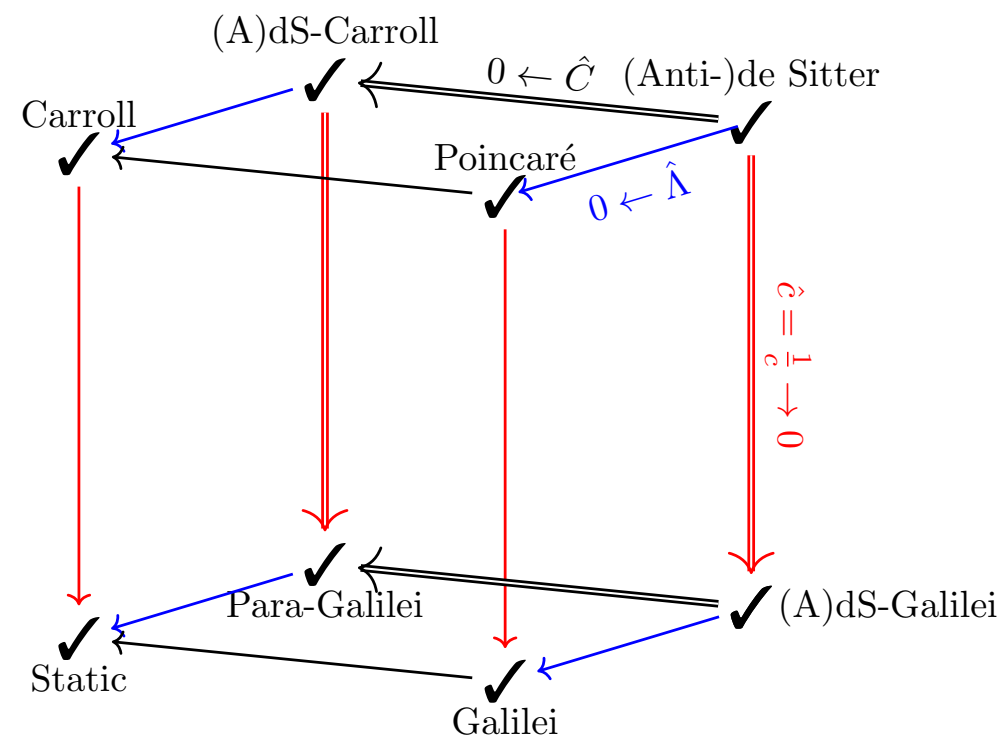

Figure 3. Kinematical limits of (anti-)de Sitter: non-relativistic/Galilean $\left(\hat{c}=\frac{1}{c} \rightarrow 0\right)$; flat $(\hat{\Lambda} \rightarrow 0)$; ultra-relativistic/Carrollian $(\hat{C} \rightarrow 0)$. Arrows with two lines imply there are two different limits, depending on the sign of the cosmological constant.

see figure 3. To reduce clutter, the central extension $M$ was dropped in this diagram and the Euclidean cases and the light cone algebra of the previous section, that does not follow from any limit, are not represented in this diagram.

The new generator $\mathrm{M}$ for $\hat{c} \neq 0$ is a trivial central extension that before taking any limits could be eliminated by a shift of $\mathrm{H}$, which shows that the starting point is actually the direct sum $\mathfrak{s l}(2, \mathbb{R}) \oplus \mathfrak{u}(1)$. However, it becomes a nontrivial central extension in the Galilean limit $\hat{c} \rightarrow 0$. We refer to the centrally extended Galilean algebra with nonzero $\hat{\Lambda}$ as the extended (A)dS-Galilei algebra (also sometimes referred to as the Newton-Hooke algebra). Sending $\hat{\Lambda} \rightarrow 0$ leads to the centrally extended Galilean algebra, better known as the Bargmann algebra. We could have added a similar central extension on the right hand side of each of the other brackets leading to centrally extended Carrollian and Poincaré algebras.

The contracted action, equations of motion and gauge symmetries are well defined as long as the Lie algebra contraction is well defined. We have summarized further possibly interesting algebras that do not follow from a kinematical limit, like Lifshitz, Schrödinger and $1 / c$ expanded Poincaré in appendix B.

We now construct the limits of the action, equations of motion, and gauge transformations explicitly and study their Lorentzian, Galilean and Carrollian invariants. The coadjoint scalar $\mathcal{X}^{*}$ and the gauge connection $A$ are parametrized as

$$
\mathcal{X}^{*}=X \mathrm{~B}^{*}+X_{\mathrm{H}} \mathrm{H}^{*}+X_{\mathrm{P}} \mathrm{P}^{*}+X_{\mathrm{M}} \mathrm{M}^{*} \quad A=\omega \mathrm{B}+e^{a} \mathrm{P}_{a}+m \mathrm{M}=\omega \mathrm{B}+\tau \mathrm{H}+e \mathrm{P}+m \mathrm{M}
$$

where the dual basis is defined by

$$
\mathrm{B}^{*}(\mathrm{~B})=1 \quad \mathrm{H}^{*}(\mathrm{H})=1 \quad \mathrm{P}^{*}(\mathrm{P})=1 \quad \mathrm{M}^{*}(\mathrm{M})=1 .
$$


The Lagrangian is given by

$$
\mathscr{L}\left[\mathcal{X}^{*}, A\right]=X F(\mathrm{~B})+X_{\mathrm{H}} F(\mathrm{H})+X_{\mathrm{P}} F(\mathrm{P})+X_{\mathrm{M}} F(\mathrm{M})
$$

with curvature

$$
\begin{aligned}
F & =F(\mathrm{~B}) \mathrm{B}+F(\mathrm{H}) \mathrm{H}+F(\mathrm{P}) \mathrm{P}+F(\mathrm{M}) \mathrm{M} \\
& =(d \omega-\hat{\Lambda} \tau \wedge e) \mathrm{B}+\left(d \tau+\hat{c}^{2} \omega \wedge e\right) \mathrm{H}+\left(d e \mp \hat{C}^{2} \omega \wedge \tau\right) \mathrm{P}+(d m+\alpha \omega \wedge e) \mathrm{M} .
\end{aligned}
$$

The action is invariant under the gauge transformations parametrized by $\lambda=\lambda^{\mathrm{B}} \mathrm{B}+\lambda^{\mathrm{H}} \mathrm{H}+$ $\lambda^{\mathrm{P}} \mathrm{P}+\lambda^{\mathrm{M}} \mathrm{M}$,

$$
\begin{aligned}
\delta_{\lambda} A= & {\left[-d \lambda^{\mathrm{B}}+\hat{\Lambda}\left(\tau \lambda^{\mathrm{P}}-e \lambda^{\mathrm{H}}\right)\right] \mathrm{B}+\left[-d \lambda^{\mathrm{H}}-\hat{c}^{2}\left(\omega \lambda^{\mathrm{P}}-e \lambda^{\mathrm{B}}\right)\right] \mathrm{H} } \\
& +\left[-d \lambda^{\mathrm{P}} \pm \hat{C}^{2}\left(\omega \lambda^{\mathrm{H}}-\tau \lambda^{\mathrm{B}}\right)\right] \mathrm{P}+\left[-d \lambda^{\mathrm{M}}-\alpha\left(\omega \lambda^{\mathrm{P}}-e \lambda^{\mathrm{B}}\right)\right] \mathrm{M} \\
\delta_{\lambda} \mathcal{X}^{*}= & {\left[\mp \hat{C}^{2} X_{\mathrm{P}} \lambda^{\mathrm{H}}+\left(\alpha X_{\mathrm{M}}+\hat{c}^{2} X_{\mathrm{H}}\right) \lambda^{\mathrm{P}}\right] \mathrm{B}^{*}+\left[ \pm \hat{C}^{2} X_{\mathrm{P}} \lambda^{\mathrm{B}}-\hat{\Lambda} X \lambda^{\mathrm{P}}\right] \mathrm{H}^{*} } \\
& +\left[\hat{\Lambda} X \lambda^{\mathrm{H}}-\left(\alpha X_{\mathrm{M}}+\hat{c}^{2} X_{\mathrm{H}}\right) \lambda^{\mathrm{B}}\right] \mathrm{P}^{*} .
\end{aligned}
$$

All contraction parameters have positive exponent and consequently the limits are welldefined. The equations of motion of (3.13) are $F=0$ and

$$
\begin{aligned}
d \mathcal{X}^{*}+\operatorname{ad}_{A}^{*} \mathcal{X}^{*}= & {\left[d X \mp \hat{C}^{2} X_{\mathrm{P}} \tau+\left(\alpha X_{\mathrm{M}}+\hat{c}^{2} X_{\mathrm{H}}\right) e\right] \mathrm{B}^{*}+\left[d X_{\mathrm{H}} \pm \hat{C}^{2} X_{\mathrm{P}} \omega-\hat{\Lambda} X e\right] \mathrm{H}^{*} } \\
& +\left[d X_{\mathrm{P}}+\hat{\Lambda} X \tau-\left(\alpha X_{\mathrm{M}}+\hat{c}^{2} X_{\mathrm{H}}\right) \omega\right] \mathrm{P}^{*}+d X_{\mathrm{M}} \mathrm{M}^{*}=0
\end{aligned}
$$

Since they provide us with additional geometric information, we first investigate the invariants of the local boosts

$$
\delta_{\lambda^{\mathrm{B}}} \tau=\hat{c}^{2} \lambda^{\mathrm{B}} e \quad \delta_{\lambda^{\mathrm{B}}} e=\mp \hat{C}^{2} \lambda^{\mathrm{B}} \tau .
$$

Expectedly, these transformations are independent of curvature, as evident from their independence of the parameter $\hat{\Lambda}$. Without taking any limit neither $\tau$ nor $e$ is invariant, which is familiar from Lorentzian geometry where no distinguished invariant vector field or one-form exists. In the Galilean limit $\hat{c} \rightarrow 0$ we get the invariant 'clock one-form' $\tau$. In the Carrollian limit the spatial zweibein component $e$ is invariant. Only in the Lorentzian/Euclidean case we can define the invariant Lorentzian/Euclidean non-degenerate metric

$$
g=\mp \tau^{2}+e^{2}
$$

This is a manifestation of the fact that we are looking beyond Lorentzian geometries, and it justifies our claim to define Galilean and Carrollian gravitational theories. Additionally this shows that theories based on the same algebra can be geometrically different, but are connected via dualities upon exchanging, e.g., time and space as in the case of Galilei and Carroll. This should be viewed as a local statement, since globally the spatial direction may be compact and the time direction non-compact. 
We will study the equations of motion of the Lagrangian (3.13) for the special cases $\hat{c}=0$ or $\hat{C}=0$. When $\hat{c}$ and $\hat{C}$ are both nonzero the equations setting the $\mathrm{H}$ and $\mathrm{P}$ curvatures equal to zero are the zero torsion conditions in the Cartan description of Euclidean/Lorentzian geometries. The zero torsion equations can be solved for $\omega$. The abelian curvature of $\omega$, i.e., $d \omega$, is then either set equal to the volume form (for $\hat{\Lambda} \neq 0$ ) or to zero (for $\hat{\Lambda}=0$ ), corresponding to the usual $1+1$ dimensional maximally symmetric Euclidean/Lorentzian spaces. There is also a zero curvature abelian gauge field, namely $m-\frac{\alpha}{\hat{c}^{2}} \tau$.

When $\hat{C}=0$ we are dealing with the following curvature equations

$$
\begin{array}{r}
d \tau+\hat{c}^{2} \omega \wedge e=0 \\
d e=0 \\
d m+\alpha \omega \wedge e=0 \\
d \omega-\hat{\Lambda} \tau \wedge e=0
\end{array}
$$

which correspond to a constant curvature 2-dimensional Carrollian geometry with a zero curvature $\mathrm{U}(1)$ gauge field on it that is given by $m-\frac{\alpha}{\hat{c}^{2}} \tau$. The fact that $d e=0$ can be interpreted as vanishing extrinsic curvature. To see this, one evaluates the 2 -form de on the two vectors that are dual to the 1 -forms $\tau$ and $e$. This leads to an expression involving the Lie derivative of the Carrollian metric ee along the vector $v$ that spans the kernel of ee (see $[53,54]$ for a general discussion of 2-dimensional Carrollian geometries). The vanishing of $d e=0$ can also be rephrased as saying that the intrinsic torsion is zero [55]. Unlike in the Lorentzian case, for Carrollian geometries we cannot solve for $\omega$ in terms of the vielbeine (and possibly the $m$ connection), since the curvature equations do not fix the vielbein component of $\omega$ along $e$, which is thus an independent field. This is not uncommon for Carrollian geometries. For example in [56] the undetermined components of $\omega$ were shown to correspond to Lagrange multipliers enforcing the constraint that the extrinsic curvature vanishes. In our setting the vanishing of the extrinsic curvature results from varying $X_{\mathrm{P}}$ in the Lagrangian. The role of $\omega$ is to ensure that the curvatures are Carroll boost invariant.

We next consider the case $\hat{c}=0$, setting $\hat{C}=\alpha=1$ without loss of generality. In this case the $F=0$ equations of motion read

$$
\begin{aligned}
d \tau & =0 \\
d e \mp \omega \wedge \tau & =0 \\
d m+\omega \wedge e & =0 \\
d \omega-\hat{\Lambda} \tau \wedge e & =0 .
\end{aligned}
$$

We interpret these equations in the language of Newton-Cartan (NC) geometry. The second and third equation are the curvature constraints imposed to be able to fully solve for the boost connection $\omega$ in terms of the NC fields $\tau, e$ and $m$ [57]. The remaining two equations fix the NC geometry. The top equation states that the clock one-form $\tau$ is closed and so these Newton-Cartan spaces admit absolute time (provided there are no closed time circles 
so that $\tau$ is exact). The last equation fixes the boost curvature which can be viewed as the $1+1$ dimensional version of the equation for $\mathrm{NC}$ gravity. It is interesting to point out that in $2 \mathrm{~d}$ we can formulate a Lagrangian theory of $\mathrm{NC}$ gravity (coupled to scalars) whereas in $2+1$ dimensions the Chern-Simons formulation of NC gravity requires an additional connection related to a generator that is not contained within the Bargmann algebra [34, 36, 37]. The $1+1$ dimensional case with nonzero $\hat{\Lambda}$ is based on the extended (A)dS Galilei algebra, also called (extended) Newton-Hooke algebra, which in $1+1$ dimensions admits an invariant metric. The case with $\hat{\Lambda}=0$ leads to the Bargmann algebra which does not admit an invariant metric.

\subsection{Newton-Cartan dilaton gravity and Carroll dilaton gravity}

The Lagrangian (3.13) with all contraction parameters set to unity is identical to the Lagrangian of the JT model (3.4), after removing the $\mathfrak{u}(1)$ field $\mathrm{M}$ by a redefinition of the generator H. As discussed above, the Lagrangian (3.13) with $\hat{c}=0$ defines a NC geometry. It is then fair to ask what is the $\mathrm{NC}$ version of more generic dilaton gravity (3.5) (or its torsionful generalization below that equation)?

We propose that this generalization is given by the NC dilaton gravity Lagrangian

$$
\mathscr{L}_{\mathrm{NCdil}}=X d \omega+V\left(X, X_{\mathrm{H}}\right) \tau \wedge e+X_{\mathrm{H}} d \tau+X_{\mathrm{P}}(d e \mp \omega \wedge \tau)+X_{\mathrm{M}}(d m+\omega \wedge e) .
$$

where $V\left(X, X_{\mathrm{H}}\right) \propto X$ for the NC limit of JT and an arbitrary function more generally. The $\mp$ signs in front of $\omega$ in the third term can be exchanged by a field redefinition, so they do not denote a distinction between a Lorentzian or Euclidean version of NC dilaton gravity. We nevertheless keep them to show they do not matter.

Only two of the curvature equations (3.20) change, but we nevertheless display all for convenience on the left hand side below, together with the remaining half of the equations of motion on the right hand side.

$$
\begin{array}{rlrl}
d \tau+\partial_{X_{\mathrm{H}}} V\left(X, X_{\mathrm{H}}\right) \tau \wedge e & =0 & d X_{\mathrm{H}} \pm \omega X_{\mathrm{P}}+e V\left(X, X_{\mathrm{H}}\right) & =0 \\
d e \mp \omega \wedge \tau & =0 & d X_{\mathrm{P}}-\omega X_{\mathrm{M}}-\tau V\left(X, X_{\mathrm{H}}\right) & =0 \\
d m+\omega \wedge e=0 & d X_{\mathrm{M}}=0 \\
d \omega+\partial_{X} V\left(X, X_{\mathrm{H}}\right) \tau \wedge e=0 & d X \mp X_{\mathrm{P}} \tau+X_{\mathrm{M}} e=0
\end{array}
$$

The first equation on the left only changes if $V$ does depend on $X_{\mathrm{H}}$ and implies that torsion no longer vanishes, i.e., the $\mathrm{NC}$ clock 1 -form no longer locally is given by $\tau=d t$, in general. Thus, if one requires NC dilaton gravity to maintain torsionlessness the potential $V$ should depend on $X$ only, just like in (3.5). The last equation on the left controls the curvature of the geometry. For functions $V$ that are non-linear in $X$ the curvature is not constant. The equations on the right hand side are the $\mathrm{NC}$ version of the dilaton equations of motion. As in ordinary dilaton gravity (see, e.g., [58]) there is a 'constant dilaton' and a 'linear dilaton' sector.

$$
\begin{array}{lllrl}
\text { constant dilaton : } & X_{\mathrm{M}}=0=X_{\mathrm{P}} & X=X^{c} & X_{\mathrm{H}}=X_{\mathrm{H}}^{c} \\
\text { linear dilaton : } & X_{\mathrm{M}}=\text { const. } & X=X(t, x) & X_{\mathrm{P}, \mathrm{H}}=X_{\mathrm{P}, \mathrm{H}}(t, x)
\end{array}
$$


The constants $X^{c}$ and $X_{\mathrm{H}}^{c}$ are determined as roots of the potential, $V\left(X^{c}, X_{\mathrm{H}}^{c}\right)=0$. As in ordinary dilaton gravity, curvature is constant for constant dilaton solutions; by contrast, torsion does not vanish but is also constant for general constant dilaton solutions

$$
* d \omega=-\partial_{X} V\left(X, X_{\mathrm{H}}\right) \quad * d \tau=-\partial_{X_{\mathrm{H}}} V\left(X, X_{\mathrm{H}}\right) .
$$

We defined our orientation by $*(\tau \wedge e)=1$.

The Lagrangian (3.21) is invariant under a non-linear modification of the symmetries (3.15)

$$
\begin{aligned}
\delta \tau & =-d \lambda^{\mathrm{H}}-\partial_{X_{\mathrm{H}}} V\left(\tau \lambda^{\mathrm{P}}-e \lambda^{\mathrm{H}}\right) & & \delta X_{\mathrm{H}}=X_{\mathrm{P}} \lambda^{\mathrm{B}}+V \lambda^{\mathrm{P}} \\
\delta e & =-d \lambda^{\mathrm{P}} \pm \omega \lambda^{\mathrm{H}} \mp \tau \lambda^{\mathrm{B}} & & \delta X_{\mathrm{P}}=-V \lambda^{\mathrm{H}}-X_{\mathrm{M}} \lambda^{\mathrm{B}} \\
\delta m & =-d \lambda^{\mathrm{M}}-\omega \lambda^{\mathrm{P}}+e \lambda^{\mathrm{B}} & \delta X_{\mathrm{M}} & =0 \\
\delta \omega & =-d \lambda^{\mathrm{B}}-\partial_{X} V\left(\tau \lambda^{\mathrm{P}}-e \lambda^{\mathrm{H}}\right) & \delta X & =\mp X_{\mathrm{P}} \lambda^{\mathrm{H}}+X_{\mathrm{M}} \lambda^{\mathrm{P}} .
\end{aligned}
$$

Choosing a field-dependent parametrization of the gauge transformations, $\lambda=\xi \cdot A+\lambda^{\mathrm{B}} \mathrm{B}+$ $\lambda^{\mathrm{M}} \mathrm{M}$, where $\xi$ is a $2 \mathrm{~d}$ vector field, establishes that gauge transformations reduce on-shell to diffeomorphisms generated by $\xi$, Galilean boosts $\lambda^{\mathrm{B}}$, and $\mathfrak{u}(1)$ transformations $\lambda^{\mathrm{M}}$.

The general solution of NC dilaton gravity in the linear dilaton sector is obtained as follows [for simplicity we restrict to the torsionless case $V=V(X)$ ]. First we solve the clock 1-form equation $d \tau=0$ by $\tau=d t$, which partly gauge fixes diffeomorphisms. Next, we gauge fix boost invariance by demanding $e=K(t, x) d x$. Most of the remaining diffeomorphism invariance is fixed by setting $K(t, x)=1$. Finally, there is another abelian gauge symmetry generated by $\lambda^{\mathrm{M}}$ that we exploit to fix the mass 1 -form as $m=\Phi(t, x) d t$, where $\Phi$ is interpreted as Newton potential. At this stage the residual gauge transformations are trivial coordinate shifts, $t \rightarrow t+t_{0}$ and $x \rightarrow x+x_{0}$, and time-dependent shifts generated by $\mathfrak{u}(1)$ transformations with $\lambda_{\mathrm{M}}=f(t)$ and boosts accompanied by compensating spatial diffeomorphisms with $\lambda_{\mathrm{B}}=\mp \partial_{t} \xi^{x}(t)=g(t)$. The latter two can be used to fix to zero integration functions encountered below.

Our 1-forms read

$$
\tau=d t \quad e=d x \quad m=\Phi(t, x) d t \quad \omega=\partial_{x} \Phi(t, x) d t .
$$

The result (3.27) solves all the equations of motion on the left side of (3.22) provided the Newton potential $\Phi$ obeys the second order partial differential equation

$$
\partial_{x}^{2} \Phi(t, x)=\frac{d V(X)}{d X}
$$

If $V=$ const. we are back to the $\mathrm{NC}$ case with cosmological constant and obtain the expected confining potential, $\Phi \propto x$, after setting to zero all integration functions by residual gauge fixing.

The right half of the equations of motion (3.22) is solved as follows. The penultimate one trivially yields $X_{\mathrm{M}}=-1 / m_{0}=$ const. The other three equations combined yield the non-linear (Casimir) relation

$$
X_{\mathrm{M}} X_{\mathrm{H}} \pm \frac{1}{2} X_{\mathrm{P}}^{2}=w(X) \quad w(X):=\int^{X} V(y) d y .
$$


The last equation of motion on the right (3.22) integrates to $X=x / m_{0}+x_{0}(t)$ where $\dot{x}_{0}(t)= \pm X_{\mathrm{P}}(t)$. We exploit now the residual gauge transformation generated by $\lambda_{\mathrm{B}}=$ $\mp \partial_{t} \xi^{x}(t)=g(t)$ to gauge fix $X_{\mathrm{P}}=0$, yielding $\partial_{X} \Phi=V$ when plugged into the second equation of motion on the right (3.22). Integrating this equation yields $\Phi=w(X)+$ $\Phi_{0}(t)$, where the integration function $\Phi_{0}$ can be gauge fixed to zero with our remaining residual gauge transformation generated by $\lambda_{\mathbb{M}}=f(t)$. Finally, the relation (3.29) can be solved for $X_{\mathrm{H}}$.

Our solution

$$
X=\frac{x}{m_{0}} \quad X_{\mathrm{M}}=-1 \quad X_{\mathrm{P}}=0 \quad X_{\mathrm{H}}=-m_{0} w(X)
$$

shows that the label 'linear dilaton sector' is indeed justified, as the dilaton is linear in the spatial coordinate $x$. It contains one relevant constant of motion, $m_{0}$, related to the mass of the solution. In the chosen gauge the Newton potential

$$
\Phi(X)=w(X)
$$

depends only on the spatial coordinate $x$ and, via $X$, also on the mass parameter $m_{0}$.

To give one example we choose $V(X)=1 / X^{2}$, obtaining the solution above with

$$
\Phi(x)=-\frac{m_{0}}{x}
$$

which is just the Newton-potential in three spatial dimensions for an object of mass $m_{0}$. This shows that one can obtain higher-dimensional Newton potentials by choosing $V(X)$ suitably, which is again identical to how things work in usual $2 \mathrm{~d}$ dilaton gravity.

Along the same lines we also propose general Carrollian dilaton gravity

$$
\mathscr{L}_{\text {Car-dil }}=X d \omega+V\left(X, X_{\mathrm{P}}\right) \tau \wedge e+X_{\mathrm{H}}(d \tau+\omega \wedge e)+X_{\mathrm{P}} d e .
$$

Here we eliminated the $\mathfrak{u}(1)$ field by a redefinition of $\mathrm{H}$. The $X$-dependence of the new potential term $V$ determines the curvature of the geometry with linear $X$-dependence corresponding to constant curvature. A non-trivial $X_{\mathrm{P}}$-dependence on the other hand leads to non-vanishing $d e$ and thus controls the extrinsic curvature or intrinsic torsion of the geometry as discussed in section 3.3. The discussion of equations of motion, constant and linear dilaton sectors is analogous to the $\mathrm{NC}$ case above.

$$
\begin{array}{lllr}
\text { constant dilaton : } & X_{\mathrm{H}}=0 & X=X^{c} & X_{\mathrm{P}}=X_{\mathrm{P}}^{c} \\
\text { linear dilaton : } & X_{\mathrm{H}}=X_{\mathrm{H}}(t, x) & X=X(t, x) & X_{\mathrm{P}, \mathrm{H}}=X_{\mathrm{P}, \mathrm{H}}(t, x)
\end{array}
$$

The constants $X^{c}$ and $X_{\mathrm{P}}^{c}$ are again determined as roots of the potential, $V\left(X^{c}, X_{\mathrm{P}}^{c}\right)=0$, and curvature is again constant in the constant dilaton sector, $* d \omega=-\partial_{X} V$ (the same is true for $\left.* d e=-\partial_{X_{\mathrm{P}}} V\right)$. 


\section{Metric BF theories and their limits}

For metric $\mathrm{BF}$ theories we demand the existence of an invariant metric on the gauge algebra. In that case taking limits, especially of the invariant metric of the Lie algebra, is more subtle since the contracted Lie algebras do not necessarily inherit the non-degeneracy of the metric. Since many interesting theories are based on metric Lie algebras and this additional structure plays a rôle for our setup of boundary conditions, we explain first when the limit has the chance to lead to another metric BF theory. Then we show how the algebras and limits can be generalized to also obtain Poincaré, Carrollian and Galilean BF theories and observe the relation of the latter to NC theory. Before discussing boundary conditions in the next section we provide a summary of the various theories we have unveiled at the end of this section.

\subsection{Metrics and limits}

Starting point is the decomposed (A)dS algebra (3.1)

$$
[\mathrm{B}, \mathrm{H}]=\mp \hat{C}^{2} \mathrm{P} \quad[\mathrm{B}, \mathrm{P}]=\hat{c}^{2} \mathrm{H} \quad[\mathrm{H}, \mathrm{P}]=-\hat{\Lambda} \mathrm{B}
$$

with the invariant metric

$$
\langle\mathrm{B}, \mathrm{B}\rangle= \pm \mu \hat{c}^{2} \hat{C}^{2} \quad\langle\mathrm{H}, \mathrm{H}\rangle= \pm \mu \hat{C}^{2} \hat{\Lambda} \quad\langle\mathrm{P}, \mathrm{P}\rangle=\mu \hat{c}^{2} \hat{\Lambda}
$$

parametrized such that any limit of the Lie algebra is well defined on the level of the Lie algebra and metric. Taking either the flat $(\hat{\Lambda} \rightarrow 0)$, Galilean $\left(\hat{c}=\frac{1}{c} \rightarrow 0\right)$ or Carrollian $(\hat{C} \rightarrow 0)$ limit of $(4.2)$ leads to a degenerate metric. In case we want to end up with a metric BF theory there exists a necessary condition

$$
\operatorname{dim} \mathfrak{g}=\operatorname{dim}[\mathfrak{g}, \mathfrak{g}]+\operatorname{dim} Z(\mathfrak{g})
$$

for the existence of a metric on a Lie algebra $\mathfrak{g}$ where $Z(\mathfrak{g})$ is the center of the Lie algebra (see, e.g., section 3.2 in [39]). As long as we do not take any limit, the center is trivial and $3=3+0$. However, taking any limit reduces $\operatorname{dim}[\mathfrak{g}, \mathfrak{g}]$ by one without adding any element to the center, i.e., $3 \neq 2+0$ and no invariant metric is possible. The addition of a nontrivial central element adds one element to the dimension of the Lie algebra (on the left hand side), but two on the right hand side, balancing the equation again, $3+1=(2+1)+(0+1)$.

In the following sections we show that the addition of central extensions is also sufficient to equip the Lie algebras and theories with an invariant metric and show how these theories can be obtained from a limit. Another option to obtain algebras with a non-degenerate invariant metric is based on so called coadjoint Lie algebras as discussed in appendix C.

\subsection{Flat space dilaton gravity}

A metric BF formulation for the Poincaré algebra is only possible when the algebra is suitably centrally extended. We show now how to obtain the resulting theory as a limit of (A)dS at the level of the action. 
We start with the (A)dS algebra (3.1) and add an additional generator M such that the algebra is given by

$$
\left[\mathrm{B}, \mathrm{P}_{a}\right]=-\epsilon_{a}{ }^{b} \mathrm{P}_{b} \quad\left[\mathrm{P}_{a}, \mathrm{P}_{b}\right]=\epsilon_{a b}(-\hat{\Lambda} \mathrm{B}+\mathrm{M}) .
$$

At this point $\mathrm{M}$ is still a trivial central extension, but it is introduced such that after the flat limit $\hat{\Lambda} \rightarrow 0$ it is nontrivial and leads to an invariant metric for (centrally extended) Poincaré. The well-defined limit on the Lie algebra implies upon substitution of the fields a limit of the equations of motion.

It remains to show that we can also take the limit at the level of the action, for which we introduce the shifted invariant metric (cf. (3.2))

$$
\begin{aligned}
\langle\mathrm{B}, \mathrm{B}\rangle & =\frac{\mu+\mu_{2}}{\hat{\Lambda}} & \left\langle\mathrm{P}_{a}, \mathrm{P}_{b}\right\rangle & =\mu \eta_{a b} \\
\langle\mathrm{M}, \mathrm{M}\rangle & =\mu_{2} \hat{\Lambda} & \langle\mathrm{B}, \mathrm{M}\rangle & =\mu_{2}
\end{aligned}
$$

where we used $\mu \mapsto \frac{\mu}{\hat{\Lambda}}$. We now take the flat limit $\hat{\Lambda} \rightarrow 0$, assuming $\chi=\left(\mu+\mu_{2}\right) / \hat{\Lambda}$ remains finite, yielding

$$
\left[\mathrm{B}, \mathrm{P}_{a}\right]=-\epsilon_{a}{ }^{b} \mathrm{P}_{b} \quad\left[\mathrm{P}_{a}, \mathrm{P}_{b}\right]=\epsilon_{a b} \mathrm{M}
$$

with invariant metric

$$
\left\langle\mathrm{P}_{a}, \mathrm{P}_{b}\right\rangle=\mu \eta_{a b} \quad\langle\mathrm{~B}, \mathrm{M}\rangle=-\mu \quad\langle\mathrm{B}, \mathrm{B}\rangle=\chi .
$$

This is the (non-semisimple) extended Poincaré algebra with non-degenerate metric for $\mu \neq 0$. This means we can write down a metric BF theory (2.4), which yields the CGHS model [31] in the formulation of Cangemi and Jackiw [59] (see also [60, 61]).

The algebra (4.6) has a higher dimensional generalization, called Maxwell algebra, which emerges in the study of particles in classical homogeneous electromagnetic fields [62, 63]. This is also true for the extended Poincaré (Euclidean) algebra which arise when considering a charged particle in $1+1$ dimensions in a constant electric (magnetic) field. From the point of view of metric Lie algebras the Maxwell algebra is the natural metric generalization of Poincaré [39].

The Carrollian and Galilean limits can be done analogously to the flat limit above. They lead to (A)dS Carroll and (A)dS Galilei theories, respectively, and are related to the CGHS model via geometric dualities as summarized in table 1.

\subsection{Summary}

We have summarized interesting homogeneous spaces and Lie algebras in table 1, which is best read together with figure 3 .

The table starts by providing the necessary information to construct the theories based on the simple Lie algebras $\mathfrak{s l}(2, \mathbb{R}) \simeq \mathfrak{s o}(2,1) \simeq \mathfrak{s o}(1,2)$ and $\mathfrak{s o}(3)$. They encompass the well known (A)dS BF theories, their Euclidean cousins and the light cone BF theory of section 3.2. Table 1 makes explicit that all, but the sphere, are based on the same Lie 


\begin{tabular}{|c|c|c|c|c|c|c|c|c|c|c|c|}
\hline \multirow{2}{*}{$\begin{array}{l}\text { Homogeneous space } \\
\text { Sphere }\end{array}$} & \multirow[t]{2}{*}{$\begin{array}{l}\text { Limit } \\
(\rightarrow 0)\end{array}$} & \multicolumn{5}{|c|}{ Nonzero commutation relations } & \multirow{2}{*}{\begin{tabular}{|c|}
$\mathrm{ND} ?$ \\
$\checkmark$
\end{tabular}} & \multicolumn{4}{|c|}{ Invariant metric } \\
\hline & & $\mathfrak{s o}(3)$ & IX & {$[B, H]=-P$} & {$[B, P]=H$} & {$[\mathrm{H}, \mathrm{P}]=-\mathrm{B}$} & & $\langle\mathrm{B}, \mathrm{B}\rangle=\mu$ & $\langle\mathrm{H}, \mathrm{H}\rangle=\mu$ & $\langle\mathrm{P}, \mathrm{P}\rangle=\mu$ & \\
\hline de Sitter (dS) & & $\mathfrak{s o}(2,1)$ & VIII & {$[\mathrm{B}, \mathrm{H}]=\mathrm{P}$} & {$[B, P]=H$} & {$[\mathrm{H}, \mathrm{P}]=-\mathrm{B}$} & $\checkmark$ & $\langle\mathrm{B}, \mathrm{B}\rangle=-\mu$ & $\langle\mathrm{H}, \mathrm{H}\rangle=-\mu$ & $\langle\mathrm{P}, \mathrm{P}\rangle=\mu$ & \\
\hline Hyperbolic & & $\mathfrak{s o}(2,1)$ & VIII & {$[\mathrm{B}, \mathrm{H}]=-\mathrm{P}$} & {$[B, P]=H$} & {$[\mathrm{H}, \mathrm{P}]=\mathrm{B}$} & $\checkmark$ & $\langle\mathrm{B}, \mathrm{B}\rangle=\mu$ & $\langle\mathrm{H}, \mathrm{H}\rangle=-\mu$ & $\langle\mathrm{P}, \mathrm{P}\rangle=-\mu$ & \\
\hline Anti-de Sitter (AdS) & & $\mathfrak{s o}(1,2)$ & VIII & {$[\mathrm{B}, \mathrm{H}]=\mathrm{P}$} & {$[\mathrm{B}, \mathrm{P}]=\mathrm{H}$} & {$[\mathrm{H}, \mathrm{P}]=\mathrm{B}$} & $\checkmark$ & $\langle\mathrm{B}, \mathrm{B}\rangle=-\mu$ & $\langle\mathrm{H}, \mathrm{H}\rangle=\mu$ & $\langle\mathrm{P}, \mathrm{P}\rangle=-\mu$ & \\
\hline Light cone & & $\mathfrak{s l}(2, \mathbb{R})$ & VIII & {$[\mathrm{B}, \mathrm{H}]=-\mathrm{B}$} & {$[\mathrm{B}, \mathrm{P}]=\mathrm{H}$} & {$[\mathrm{H}, \mathrm{P}]=-\mathrm{P}$} & $\checkmark$ & $\langle\mathrm{B}, \mathrm{P}\rangle=\mu$ & $\langle\mathrm{H}, \mathrm{H}\rangle=\mu$ & & \\
\hline Euclidean & $\hat{\Lambda}$ & iso $(2)$ & $\mathrm{VII}_{0}$ & {$[\mathrm{~B}, \mathrm{H}]=-\mathrm{P}$} & {$[\mathrm{B}, \mathrm{P}]=\mathrm{H}$} & & $x$ & $\langle\mathrm{~B}, \mathrm{~B}\rangle=\mu$ & & & \\
\hline Extended Euclidean & $\hat{\Lambda}$ & $\operatorname{iso}(2)^{c}$ & $\mathrm{VII}_{0}^{c}$ & {$[\mathrm{~B}, \mathrm{H}]=-\mathrm{P}$} & {$[\mathrm{B}, \mathrm{P}]=\mathrm{H}$} & {$[\mathrm{H}, \mathrm{P}]=\mathrm{M}$} & $\checkmark$ & $\langle\mathrm{B}, \mathrm{B}\rangle=\chi$ & $\langle\mathrm{H}, \mathrm{H}\rangle=\mu$ & $\langle\mathrm{P}, \mathrm{P}\rangle=\mu$ & $\langle\mathrm{B}, \mathrm{M}\rangle=-\mu$ \\
\hline dS-Carroll & $\hat{C}$ & $\mathfrak{i s o}(2)$ & $\mathrm{VII}_{0}$ & & {$[\mathrm{~B}, \mathrm{P}]=\mathrm{H}$} & {$[\mathrm{H}, \mathrm{P}]=-\mathrm{B}$} & $x$ & & & $\langle\mathrm{P}, \mathrm{P}\rangle=\mu$ & \\
\hline Extended dS-Carroll & $\hat{C}$ & $\operatorname{iso}(2)^{c}$ & $\mathrm{VII}_{0}^{c}$ & {$[\mathrm{~B}, \mathrm{H}]=\mathrm{M}$} & {$[\mathrm{B}, \mathrm{P}]=\mathrm{H}$} & {$[\mathrm{H}, \mathrm{P}]=-\mathrm{B}$} & $\checkmark$ & $\langle\mathrm{B}, \mathrm{B}\rangle=\mu$ & $\langle\mathrm{H}, \mathrm{H}\rangle=\mu$ & $\langle\mathrm{P}, \mathrm{P}\rangle=\chi$ & $\langle\mathrm{P}, \mathrm{M}\rangle=-\mu$ \\
\hline AdS-Galilei & $\hat{c}$ & $\mathfrak{i s o}(2)$ & $\mathrm{VII}_{0}$ & {$[\mathrm{~B}, \mathrm{H}]=\mathrm{P}$} & & {$[\mathrm{H}, \mathrm{P}]=\mathrm{B}$} & $x$ & & $\langle\mathrm{H}, \mathrm{H}\rangle=\mu$ & & \\
\hline Extended AdS-Galilei & $\hat{c}$ & $\mathfrak{i s o}(2)^{c}$ & $\mathrm{VII}_{0}^{c}$ & {$[\mathrm{~B}, \mathrm{H}]=\mathrm{P}$} & {$[\mathrm{B}, \mathrm{P}]=\mathrm{M}$} & {$[\mathrm{H}, \mathrm{P}]=\mathrm{B}$} & $\checkmark$ & $\langle\mathrm{B}, \mathrm{B}\rangle=\mu$ & $\langle\mathrm{H}, \mathrm{H}\rangle=\chi$ & $\langle\mathrm{P}, \mathrm{P}\rangle=\mu$ & $\langle\mathrm{H}, \mathrm{M}\rangle=-\mu$ \\
\hline Poincaré & $\hat{\Lambda}$ & iso $(1,1)$ & $\mathrm{VI}_{0}$ & {$[\mathrm{~B}, \mathrm{H}]=\mathrm{P}$} & {$[B, P]=H$} & & $x$ & $\langle\mathrm{~B}, \mathrm{~B}\rangle=-\mu$ & & & \\
\hline Extended Poincaré & $\hat{\Lambda}$ & $\operatorname{iso}(1,1)^{c}$ & $\mathrm{VI}_{0}^{c}$ & {$[\mathrm{~B}, \mathrm{H}]=\mathrm{P}$} & {$[\mathrm{B}, \mathrm{P}]=\mathrm{H}$} & {$[\mathrm{H}, \mathrm{P}]=\mathrm{M}$} & $\checkmark$ & $\langle\mathrm{B}, \mathrm{B}\rangle=\chi$ & $\langle\mathrm{H}, \mathrm{H}\rangle=-\mu$ & $\langle\mathrm{P}, \mathrm{P}\rangle=\mu$ & $\langle\mathrm{B}, \mathrm{M}\rangle=\mu$ \\
\hline AdS-Carroll & $\hat{C}$ & $\mathfrak{i s o}(1,1)$ & $\mathrm{VI}_{0}$ & & {$[\mathrm{~B}, \mathrm{P}]=\mathrm{H}$} & {$[\mathrm{H}, \mathrm{P}]=\mathrm{B}$} & $x$ & & & $\langle\mathrm{P}, \mathrm{P}\rangle=-\mu$ & \\
\hline Extended AdS-Carroll & $\hat{C}$ & $\mathfrak{i s o}(1,1)^{c}$ & $\mathrm{VI}_{0}^{c}$ & {$[\mathrm{~B}, \mathrm{H}]=\mathrm{M}$} & {$[\mathrm{B}, \mathrm{P}]=\mathrm{H}$} & {$[\mathrm{H}, \mathrm{P}]=\mathrm{B}$} & $\checkmark$ & $\langle\mathrm{B}, \mathrm{B}\rangle=\mu$ & $\langle\mathrm{H}, \mathrm{H}\rangle=-\mu$ & $\langle\mathrm{P}, \mathrm{P}\rangle=\chi$ & $\langle\mathrm{P}, \mathrm{M}\rangle=\mu$ \\
\hline dS-Galilei & $\hat{c}$ & $\operatorname{iso}(1,1)$ & $\mathrm{VI}_{0}$ & {$[\mathrm{~B}, \mathrm{H}]=\mathrm{P}$} & & {$[\mathrm{H}, \mathrm{P}]=-\mathrm{B}$} & $x$ & & $\langle\mathrm{H}, \mathrm{H}\rangle=-\mu$ & & \\
\hline Extended dS-Galilei & $\hat{c}$ & iso $(1,1)^{c}$ & $\mathrm{VI}_{0}^{c}$ & {$[\mathrm{~B}, \mathrm{H}]=\mathrm{P}$} & {$[\mathrm{B}, \mathrm{P}]=\mathrm{M}$} & {$[\mathrm{H}, \mathrm{P}]=-\mathrm{B}$} & $\checkmark$ & $\langle\mathrm{B}, \mathrm{B}\rangle=\mu$ & $\langle\mathrm{H}, \mathrm{H}\rangle=\chi$ & $\langle\mathrm{P}, \mathrm{P}\rangle=-\mu$ & $\langle\mathrm{H}, \mathrm{M}\rangle=\mu$ \\
\hline Carroll & $\hat{C}, \hat{\Lambda}$ & Heisenberg & II & & {$[\mathrm{B}, \mathrm{P}]=\mathrm{H}$} & & $x$ & $\langle\mathrm{~B}, \mathrm{~B}\rangle=\chi_{\mathrm{B}}$ & $\langle\mathrm{B}, \mathrm{P}\rangle=\chi_{\mathrm{BP}}$ & $\langle\mathrm{P}, \mathrm{P}\rangle=\chi_{\mathrm{P}}$ & \\
\hline Doubly extended Carroll & $\hat{C}, \hat{\Lambda}$ & Heisenberg ${ }^{c c}$ & $\mathrm{II}^{c c}$ & {$[\mathrm{~B}, \mathrm{H}]=\mathrm{M}$} & {$[\mathrm{B}, \mathrm{P}]=\mathrm{H}$} & {$[\mathrm{H}, \mathrm{P}]=\mathrm{Z}$} & $\checkmark$ & $\begin{array}{l}\langle\mathrm{H}, \mathrm{H}\rangle=\mu \\
\langle\mathrm{B}, \mathrm{B}\rangle=\chi_{\mathrm{B}}\end{array}$ & $\begin{array}{l}\langle\mathrm{B}, \mathrm{Z}\rangle=-\mu \\
\langle\mathrm{B}, \mathrm{P}\rangle=\chi_{\mathrm{BP}}\end{array}$ & $\begin{array}{l}\langle\mathrm{P}, \mathrm{M}\rangle=-\mu \\
\langle\mathrm{P}, \mathrm{P}\rangle=\chi_{\mathrm{P}}\end{array}$ & \\
\hline Galilei & $\hat{c}, \hat{\Lambda}$ & Heisenberg & II & {$[\mathrm{B}, \mathrm{H}]=\mathrm{P}$} & & & $x$ & $\langle\mathrm{~B}, \mathrm{~B}\rangle=\chi_{\mathrm{B}}$ & $\langle\mathrm{B}, \mathrm{H}\rangle=\chi_{\mathrm{BH}}$ & $\langle\mathrm{H}, \mathrm{H}\rangle=\chi_{\mathrm{H}}$ & \\
\hline Doubly extended Galilei & $\hat{c}, \hat{\Lambda}$ & Heisenberg ${ }^{c c}$ & $\mathrm{II}^{c c}$ & {$[\mathrm{~B}, \mathrm{H}]=\mathrm{P}$} & {$[\mathrm{B}, \mathrm{P}]=\mathrm{M}$} & {$[\mathrm{H}, \mathrm{P}]=\mathrm{Z}$} & $\checkmark$ & $\begin{aligned}\langle\mathrm{P}, \mathrm{P}\rangle & =\mu \\
\langle\mathrm{B}, \mathrm{B}\rangle & =\chi_{\mathrm{B}}\end{aligned}$ & $\begin{array}{l}\langle\mathrm{B}, \mathrm{Z}\rangle=\mu \\
\langle\mathrm{B}, \mathrm{H}\rangle=\chi_{\mathrm{BH}}\end{array}$ & $\begin{aligned}\langle\mathrm{H}, \mathrm{M}\rangle & =-\mu \\
\langle\mathrm{H}, \mathrm{H}\rangle & =\chi_{\mathrm{H}}\end{aligned}$ & \\
\hline Para-Galilei & $\hat{c}, \hat{C}$ & Heisenberg & II & & & {$[\mathrm{H}, \mathrm{P}]=\mathrm{B}$} & $x$ & $\langle\mathrm{H}, \mathrm{H}\rangle=\chi_{\mathrm{H}}$ & $\langle\mathrm{H}, \mathrm{P}\rangle=\chi_{\mathrm{HP}}$ & $\langle\mathrm{P}, \mathrm{P}\rangle=\chi_{\mathrm{P}}$ & \\
\hline Doubly extended para-Galilei & $\hat{c}, \hat{C}$ & Heisenberg ${ }^{c c}$ & $\mathrm{II}^{c c}$ & {$[\mathrm{~B}, \mathrm{H}]=\mathrm{M}$} & {$[\mathrm{B}, \mathrm{P}]=\mathrm{Z}$} & {$[\mathrm{H}, \mathrm{P}]=\mathrm{B}$} & $\checkmark$ & $\begin{array}{l}\langle\mathrm{B}, \mathrm{B}\rangle=\mu \\
\langle\mathrm{H}, \mathrm{H}\rangle=\chi_{\mathrm{H}}\end{array}$ & $\begin{array}{l}\langle\mathrm{H}, \mathrm{Z}\rangle=-\mu \\
\langle\mathrm{H}, \mathrm{P}\rangle=\chi_{\mathrm{HP}}\end{array}$ & $\begin{array}{l}\langle\mathrm{P}, \mathrm{M}\rangle=\mu \\
\langle\mathrm{P}, \mathrm{P}\rangle=\chi_{\mathrm{P}}\end{array}$ & \\
\hline Static & $\hat{c}, \hat{C}, \hat{\Lambda}$ & Abelian & I & & & & $\checkmark$ & $\begin{array}{l}\langle\mathrm{B}, \mathrm{B}\rangle=\mu_{\mathrm{B}} \\
\langle\mathrm{B}, \mathrm{H}\rangle=\chi_{\mathrm{BH}}\end{array}$ & $\begin{array}{l}\langle\mathrm{H}, \mathrm{H}\rangle=\mu_{\mathrm{H}} \\
\langle\mathrm{B}, \mathrm{P}\rangle=\chi_{\mathrm{BP}}\end{array}$ & $\begin{array}{l}\langle\mathrm{P}, \mathrm{P}\rangle=\mu_{\mathrm{P}} \\
\langle\mathrm{H}, \mathrm{P}\rangle=\chi_{\mathrm{HP}}\end{array}$ & \\
\hline
\end{tabular}

Table 1. Overview of homogeneous spaces/Klein pairs $(\mathfrak{g}, B)$, underlying Lie algebras $\mathfrak{g}$ and their invariant metric ('ND?' indicates Non-Degeneracy). The real Lie algebras are indicated by their names and according to Bianchi's classification [64, 65]. Superscripts $c$ denote nontrivial central extensions. Rows in red are Lorentzian versions of the previous row, rows in green (blue) are centrally extended once (twice) and arise as limits of $\mathfrak{s l}(2, \mathbb{R}) \oplus \mathfrak{u}(1)(\mathfrak{s l}(2, \mathbb{R}) \oplus \mathfrak{u}(1) \oplus \mathfrak{u}(1))$, see section 4.2 .

algebra; however, they differ as homogeneous spaces (under the additional assumption that we disallow an exchange of time and space) and therefore in their geometric and physical interpretation, as described in section 2.2.

Taking either one of the flat/Carrollian/Galilean limits of the (A)dS theory we arrive at Poincaré/(A)dS Carrollian/(A)dS Galilean theories. These BF theories are based on Lie algebras that do not admit an invariant metric, see section 3.3. They allow for one nontrivial central extension that renders the invariant metric non-degenerate. These (centrally) extended theories can also be obtained from a limit, shown in section 4.2.

Taking a second limit, e.g., first the flat and then the Carrollian, leads to the Carroll, Galilei and para-Galilei theories. For these theories doubly centrally extending leads from a degenerate to a non-degenerate invariant metric. Using the procedure described in section 4.2 one can show that all the theories below the first horizontal dividing line can be obtained by contraction and taking quotients starting from the parent theory $\mathfrak{s l}(2, \mathbb{R}) \oplus \mathfrak{u}(1) \oplus \mathfrak{u}(1)$. The two $\mathfrak{u}(1)$ 's correspond to central extensions that are trivial before taking limits but become non-trivial afterwards. Nevertheless, the theory still describes a standard NC/Carroll structure since the additional field associated to the $\mathfrak{u}(1)$ generator $\mathrm{Z}$, introduced in order to have a non-degenerate metric, decouples on-shell. 
Taking all three limits leads to the static case, yielding an abelian algebra, which always allows for an invariant metric, and fulfills the necessary condition (4.3) since $3=0+3$. For the static and the para-Galilei case the group action of the boosts on the homogeneous space is trivial, i.e., they do not act at all and leave points unaltered. It is then natural to quotient by them, leading to an Aristotelian algebra that is abelian in $1+1$ dimensions.

Most of our theories arise from limits of centrally extended (A)dS, but there are further interesting spaces on which one can base Lifshitz, Schrödinger, 1/c expanded Poincaré, and coadjoint theories, that we discuss in appendix B and C. Yet another generalization are theories based on the remaining kinematical homogeneous spaces that do not follow from a limit (we do not discuss the cases of torsional Galilean and S17-S20 of table 1 in [49]).

\section{Boundary actions of kinematical BF theories}

In this section we discuss boundary actions associated with the kinematical BF theories introduced in the previous sections, restricted to metric BF theories.

\subsection{Particle on group manifold}

In the case of Chern-Simons theories in three dimensions it is well-known that these theories reduce to Wess-Zumino-Witten (WZW) models on manifolds with boundaries [66, 67]. In a similar way, it can be shown that BF theories with a particular choice of boundary condition reduce to the action of a particle on the group manifold of the chosen gauge group.

The variation of the action of a metric BF theory (2.4) on a manifold with boundary reads

$$
\delta I=\int_{\mathcal{M}}(\langle\delta \mathcal{X}, F\rangle-\langle d \mathcal{X}+[A, \mathcal{X}], \delta A\rangle)+\int_{\partial \mathcal{M}}\langle\mathcal{X}, \delta A\rangle .
$$

A possible choice of boundary conditions is to take Dirichlet boundary conditions on $A$. With this choice no further boundary term is needed for a well-defined variational principle and the theory is topological without any dynamics on the boundary. If we are to interpret the connection components as zweibein and spin-connection for a gravitational theory, putting Dirichlet boundary conditions on all components of $A$ is in general too strict. ${ }^{3}$ Instead of Dirichlet boundary conditions we impose

$$
\left.\mathcal{X} d f\right|_{\partial \mathcal{M}}=\left.A\right|_{\partial \mathcal{M}}
$$

Note that we implicitly used the invariant metric here in order to write both $\mathcal{X}$ and $A$ as elements of the Lie algebra. The one-form $d f$ is assumed to be fixed on the boundary $[13,17,68,69] .{ }^{4}$ Imposing this boundary condition requires the addition of a boundary term to (2.4) such that the full action reads

$$
I[A, \mathcal{X}]=\int_{\mathcal{M}}\langle\mathcal{X}, F\rangle-\frac{1}{2} \int_{\partial \mathcal{M}} d f\langle\mathcal{X}, \mathcal{X}\rangle
$$

\footnotetext{
${ }^{3}$ This is related to the imposition of either Dirichlet or Neumann conditions on the metric (and not both).

${ }^{4}$ In [69] this boundary condition was interpreted as a Yang-Mills theory with a position-dependent coupling constant that is localized near the boundary.
} 
The integrand of the boundary term is recognized as the quadratic Casimir

$$
C=\langle\mathcal{X}, \mathcal{X}\rangle
$$

that is conserved on-shell using the right hand side of (2.5).

We introduce a coordinate system $(\rho, t)$ with $t$ being the coordinate along the boundary that is located at $\rho \rightarrow \infty$. We assume that the homogeneous space on which the gravitational theory is defined has only one boundary component, the topology of which can be either a circle or a line.

Starting from (5.3) one can integrate out the dilaton field enforcing the constraint $F=0$ which is solved locally by

$$
A=\tilde{g}^{-1} d \tilde{g}
$$

where $\tilde{g}$ are elements of the gauge group of the BF theory. In order to simplify the discussion we assume that close to the boundary the group element $\tilde{g}$ factorizes

$$
\tilde{g}=g(t) b(\rho) .
$$

Using the boundary condition (5.2) and assuming the orientation $(\rho, t)$, the action (5.3) becomes

$$
I[g]=-\frac{1}{2} \int_{\partial \mathcal{M}} d t\left(\partial_{t} f\right)^{-1}\left\langle g^{-1} \partial_{t} g, g^{-1} \partial_{t} g\right\rangle
$$

which is the action for a particle moving on the group manifold of $G$. The gauge modes $g$ thus become physical at the boundary due to the explicit breaking of gauge invariance by the boundary condition (5.2). This action is invariant under two copies of the global symmetry group, i.e., under the transformation

$$
g \mapsto \tilde{h} g h \quad \text { with constant } \tilde{h}, h \in G
$$

with corresponding left and right charges $\tilde{Q}$ and $Q$

$$
\tilde{Q}_{\tilde{A}}=\left(\partial_{t} f\right)^{-1}\left\langle\tilde{A}, \partial_{t} g g^{-1}\right\rangle \quad Q_{A}=\left(\partial_{t} f\right)^{-1}\left\langle A, g^{-1} \partial_{t} g\right\rangle \quad \tilde{A}, A \in \mathfrak{g}
$$

where $\tilde{A}$ and $A$ are the Lie algebra generators of the corresponding group elements $\tilde{h}$ and $h$, respectively. The right charges have the Poisson bracket

$$
\left\{Q_{A}, Q_{B}\right\}=Q_{[A, B]}
$$

with a similar expression for the left charges. Note, however, that the left transformations $\tilde{h}$ correspond to a redundancy in our reduction (5.5) and should therefore be thought of as being gauged, i.e., all the left charges should be set to zero on-shell. This requirement is extrinsic to the action (5.7) and is a consequence of arriving at this action from a $2 \mathrm{~d}$ bulk action. ${ }^{5}$ Taken together, the above action is the one-dimensional equivalent to the WZW action appearing at the boundary of a CS theory in three dimensions.

\footnotetext{
${ }^{5}$ Compare this to the three dimensional cases [70, 71] where the two-dimensional boundary actions also inherit a gauge symmetry corresponding to the global symmetry group.
} 
In deriving the action we have only used the boundary condition (5.2) which can be imposed for any metric BF theory. But this is not the end of the story as ultimately we want to interpret these BF theories as (non- or ultra-relativistic) theories of gravity where one might impose additional asymptotic boundary conditions on the metric or, equivalently, on vielbein and spin-connection. In other words, we are looking for a (Hamiltonian) reduction of the boundary action (5.7).

\subsection{Hamiltonian reduction of boundary action}

For many applications we are not interested in the loosest set of boundary conditions, but rather impose (physically or geometrically motivated) restrictions on the fields. A famous example is the Drinfeld-Sokolov reduction of the $\mathfrak{s l}(2, \mathbb{R})$ current algebra to the Virasoro algebra, which applied to $\mathrm{AdS}_{3}$ gravity yields Brown-Henneaux boundary conditions [72]. For $\mathrm{AdS}_{3}$ gravity several inequivalent boundary conditions were identified, e.g., [72-75]. From the point of view of the boundary WZW model these boundary conditions act as current constraints [76]. Following this procedure one finds the Alekseev-Shatashvili action as boundary theory for $\mathrm{AdS}_{3}$ with Brown-Henneaux boundary conditions [70] or the $\mathrm{BMS}_{3}$ geometric action [71] for the boundary conditions of [77] on three-dimensional flat space. Both of these theories have a flavor of hydrodynamics in the sense that their dynamics is nothing but the conservation of the stress-energy tensor (or its Carrollian analogue in the flat space case). As reviewed below, the Schwarzian action for $\mathrm{AdS}_{2}$ can be understood in a similar way.

Boundary conditions on spin-connection and vielbein translate to conditions on the gauge-connection $A$ and by the flatness condition (5.5) to constraints on $g^{-1} \partial_{t} g$. Assuming that the boundary conditions are consistent with the algebraic structure of the particle action, we can view the former as constraints on the right charges $Q_{A} \cdot{ }^{6}$ The structure of the algebra puts restrictions on the set of possible consistent constraints and thus possible boundary conditions. In what follows we make some specific assumptions about the boundary conditions that could be (and for some applications have to be) relaxed; however, they will be useful for the two examples that we provide in section 6 .

Let $Q_{A}$ be the set of right charges of the action (5.7) based on the Lie algebra $\mathfrak{g}$. A generic constraint compatible with the algebra structure on $g^{-1} \partial_{t} g$ has the form

$$
\Phi_{\gamma} \equiv\left\langle\gamma, g^{-1} \partial_{t} g\right\rangle-\langle\gamma, K\rangle=0 \quad \gamma, K \in \mathfrak{g} .
$$

The constraint thus sets the charge $Q_{\gamma}$ on-shell to some value determined by the fixed algebra element $K$. (By fixed we imply that $K$ has vanishing Poisson brackets with all functions on phase space; we assume the same for $\gamma$ ).

Using the Poisson bracket for the right charges (5.10) we calculate the Poisson bracket of the constraints

$$
\left(\partial_{t} f\right)^{-1}\left\{\Phi_{\gamma_{1}}, \Phi_{\gamma_{2}}\right\}=\Phi_{\left[\gamma_{1}, \gamma_{2}\right]}+\left\langle\left[\gamma_{1}, \gamma_{2}\right], K\right\rangle
$$

\footnotetext{
${ }^{6}$ In the case of WZW models based on simple algebras, the question of consistent sets of constraints has been analyzed in detail in [78].
} 
We note first that a new constraint is generated on the right hand side of (5.12) unless all the generators belong to a subalgebra, i.e., $\gamma_{1}, \gamma_{2} \in \Gamma$ where $[\Gamma, \Gamma] \subset \Gamma$. The nature of the constraints is now determined by the second term. If $K \in \Gamma^{\perp}$ or the subalgebra is abelian, the constraints are first-class. ${ }^{7}$ First-class constraints are the hallmark of gauge symmetry in a system and require additional constraints in the form of gauge-fixing conditions that render the first-class constraints second-class. For the type of boundary conditions we are currently interested in, we do not want to introduce (further) gauge symmetries. Therefore, we demand that the system in (5.12) be second-class.

This means we have found a way to determine consistent boundary conditions compatible with the algebraic structure of the boundary action (5.7) by looking for evendimensional non-abelian subalgebras $\Gamma$ of the gauge algebra $\mathfrak{g}$. The constant element $K$ is subsequently chosen such that $\langle K, \Gamma\rangle \neq 0$.

In summary, boundary conditions on the fields of the BF theory compatible with the universal boundary condition (5.2) lead to the action (5.7) together with a system of second class constraints. The latter can be solved directly in the action [79].

In the above we spelled out a purely algebraic way to arrive at consistent boundary condition for a metric BF theory. This is usually not the way boundary conditions for gravitational theories are conceived. Rather, one starts from a bulk perspective and chooses boundary conditions such that they allow an interesting class of bulk geometries as solutions. This is indeed the case for the BF theory on $\mathrm{AdS}_{2}$, i.e., the JT model in the second order perspective, and we will see below that these boundary conditions satisfy the above criteria. But the purely algebraic point of view presented here allows also to find boundary conditions for BF theories with Carrollian/Galilean interpretation where the geometric picture is often not as clear as in the relativistic case. In the next section we apply this procedure to two examples.

\section{Schwarzian-like theories}

In this section we consider metric BF theories based on the various (1+1)-dimensional kinematical algebras and look for boundary conditions, as discussed in the previous section, that reduce the particle action (5.7) to Schwarzian-like actions.

To show how our proposal in section 5 works, we first review the construction of the Schwarzian action for a BF theory based on the symmetry algebra of Euclidean $\mathrm{AdS}_{2}$ in section 6.1. The main new example is the construction of the boundary action for (extended) AdS-Carroll $l_{2}$ in section 6.2. Other kinematical algebras where our procedure does not work without modifications are briefly mentioned in section 6.3.

\subsection{Euclidean $\mathbf{A d S}_{2} /$ Hyperbolic plane}

The discussion in section 5.2 instructs to look for two-dimensional non-abelian subalgebras of the Euclidean AdS algebra. In the H, B, P basis there is no obvious choice, but changing

\footnotetext{
${ }^{7}$ We use here the standard terminology to refer to constraints whose Poisson brackets vanish on-shell as first class. Constraints with Poisson brackets that do not vanish on the constraint surface are called second class. From this follows immediately that second class constraints come always in even numbers.
} 
to the $\mathrm{L}_{+}, \mathrm{L}_{0}, \mathrm{~L}_{-}$basis

$$
\mathrm{L}_{+}=\mathrm{B}+\mathrm{H} \quad \mathrm{L}_{-}=\mathrm{B}-\mathrm{H} \quad \mathrm{L}_{0}=\mathrm{P}
$$

with commutation relations explicitly given by (D.1), we find the two choices $\Gamma=\left\{\mathrm{L}_{+}, \mathrm{L}_{0},\right\}$ and $\Gamma=\left\{\mathrm{L}_{-}, \mathrm{L}_{0}\right\}$. Taking the latter pair (the other one leads to the same conclusions upon redefinition of the radial coordinate) we find from (5.12) that the fixed element $K$ has to be proportional to $\mathrm{L}_{+}$in order to have non-zero inner product with $\mathrm{L}_{-}$. It can be shifted by elements of $\mathrm{L}_{0}$ and $\mathrm{L}_{-}$although the latter element has no influence on the constraints. Choosing $K=\alpha_{+} \mathrm{L}_{+}+\alpha_{0} \mathrm{~L}_{0}$ with $\alpha_{+} \neq 0$ the constraints (5.11) read

$$
\phi_{-}=\left\langle\mathrm{L}_{-}, g^{-1} \partial_{t} g\right\rangle-2 \alpha_{+} \quad \phi_{0}=\left\langle\mathrm{L}_{0}, g^{-1} \partial_{t} g\right\rangle+\alpha_{0}
$$

which yields

$$
\left.g^{-1} \partial_{t} g\right|_{0}=\left.\alpha_{0} \quad g^{-1} \partial_{t} g\right|_{+}=\alpha_{+} .
$$

Assuming that the radial dependence of the connection (6.4) is completely captured by the group element $b=\exp \left(\rho \mathrm{L}_{0}\right)$, these constraints reduce to those implied by the well-known boundary conditions for the connection on Euclidean $\mathrm{AdS}_{2}$ (hyperbolic in table 1) for some particular choice of $\alpha_{0}$ and $\alpha_{+}$, that is ultimately inconsequential for the reduced action. In the first order formulation these boundary conditions in the highest-weight gauge (cf., e.g., $[68,80])$ are given by

$$
A_{t}=e^{\rho} \mathrm{L}_{+}+\mathcal{L}(t) e^{-\rho} \mathrm{L}_{-} \quad A_{\rho}=\mathrm{P}
$$

with the generators (4.1) and the cosmological constant set to unity. In the second order formulation these boundary conditions translate to the metric

$$
d s^{2}=d \rho^{2}+\frac{1}{4}\left(e^{\rho}-\mathcal{L}(t) e^{-\rho}\right)^{2} d t^{2}
$$

where $\mathcal{L}(t)$ is an arbitrary function and the dilaton field has the asymptotic behavior

$$
X=e^{\rho} \bar{X}+O\left(e^{-\rho}\right) .
$$

We have therefore reconstructed the above boundary conditions on $\mathrm{AdS}_{2}$ out of the algebraic considerations of section 5.2 .

Under gauge transformations generated by $\frac{1}{2} \lambda \mathrm{L}_{+}+\lambda^{0} \mathrm{~L}_{0}+\lambda^{-} \mathrm{L}_{-}$preserving the form of $A_{t}$, the field $\mathcal{L}(t)$ transforms with an infinitesimal Schwarzian derivative,

$$
\delta_{\lambda} \mathcal{L}(t)=\lambda \mathcal{L}^{\prime}+2 \lambda^{\prime} \mathcal{L}+\lambda^{\prime \prime \prime}
$$

which corresponds to the transformation of a stress tensor under infinitesimal conformal transformations. The zero-mode of the function $\mathcal{L}$ is related to the mass of spacetime [80].

We turn now to the derivation of the boundary action that follows from the particle action (5.7) upon introducing the constraints implied by the above boundary conditions. Using Gauss parametrization ${ }^{8}$ for the $t$-dependent group element

$$
g=e^{y \mathrm{~L}_{+}} e^{y^{0} \mathrm{~L}_{0}} e^{y^{-} \mathrm{L}_{-}}
$$

\footnotetext{
${ }^{8}$ This parametrization is valid for $\mathrm{SO}(2,1)=\operatorname{PSL}(2, \mathbb{R})=\operatorname{SL}(2, \mathbb{R}) /\{ \pm 1\}$ which is the group for which globally $\mathrm{AdS}_{2}$ is single valued in the first order formulation.
} 
the two constraints (6.3) lead to two algebraic equations that can be solved in terms of the field $y$. Plugging the solutions into the action (5.7) yields

$$
I[y]=\frac{1}{2} \int d t\left(\partial_{t} f\right)^{-1}\{y, t\} .
$$

where $\{\cdot, \cdot\}$ denotes the Schwarzian derivative. Using the boundary condition (5.2) we can further relate $\partial_{t} f$ to the leading order of the dilaton so that we find

$$
I[y]=\bar{X} \int d t\{y, t\}
$$

thus reproducing the Schwarzian action. The action is invariant under the finite $\operatorname{PSL}(2, \mathbb{R})$ transformations

$$
y \mapsto \frac{a y+b}{c y+d} \quad a d-b c=1
$$

that correspond to group multiplication from the left, $\tilde{h}$ in (5.8). As argued in section 6, this transformation should be thought of as a gauge symmetry.

In terms of the group elements $g$ the function $\mathcal{L}(t)$ reads

$$
\mathcal{L}(t)=-\frac{\{y, t\}}{2} .
$$

Since the equation of motion of the Schwarzian action is just

$$
\partial_{t}\{y, t\}=0
$$

we see, as in the higher-dimensional cases mentioned in section 5.1, that the Schwarzian action just encodes mass conservation.

In deriving the action (6.10) we have not assumed anything about the topology of the boundary. This is therefore the appropriate result if the boundary is taken to be a line, i.e., the zero temperature result since the boundary coordinate corresponds to Euclidean time. The finite temperature result is obtained if the boundary is taken to be a circle with periodicity $t \sim t+\beta$. Going once around the circle, the group element $g$ has to obey

$$
g(t+\beta)=g(t)
$$

in order to be single-valued. It is straightforward to see that this identification can be achieved by the field redefinition

$$
y=\tan \left(\frac{\pi h(t)}{\beta}\right)
$$

under which the Schwarzian action reads

$$
I[h]=\bar{X} \int d t\left(\{h, t\}+\frac{2 \pi^{2}}{\beta^{2}}\left(h^{\prime}\right)^{2}\right) .
$$

From the point of view taken in this section, all homogeneous spaces based on the algebra $\mathfrak{s l}(2, \mathbb{R})$ can be equipped with the above boundary conditions that lead to a Schwarzian action on the respective boundaries, modulo possible subtleties concerning the topology of the bulk spacetime; see e.g., [81] for the $\mathrm{dS}_{2}$ case. ${ }^{9}$

\footnotetext{
${ }^{9}$ While the case of the two-dimensional light cone of section 3.2 has not been worked out in detail, it is highly suggestive in light of the results $[82,83]$.
} 


\subsection{AdS-Carroll 2}

We turn our attention to a non-Lorentzian spacetime. As apparent from table 1, the algebra of extended AdS-Carroll (AdSC) is isomorphic to the centrally-extended 2d Poincaré algebra. The interpretation of the generators, however, when viewed as a homogeneous space is different. We expand the (co)adjoint vector $\mathcal{X}$ and gauge field as

$$
\mathcal{X}=X^{0} \mathrm{H}+Z \mathrm{P}+X \mathrm{~B}+X^{1} \mathrm{M} \quad A=e^{0} \mathrm{H}+e^{1} \mathrm{P}+\omega \mathrm{B}+Y \mathrm{M}
$$

where the components of the former are labelled such that $X^{0}$ enforces the torsion constraint for $e^{0}$ and so forth. In contrast to the (Euclidean) $\mathrm{AdS}_{2}$ case above, boundary condition for this spacetime have not been discussed in the literature. We therefore turn to the algebraic algorithm presented in section 5.2 in order to look for consist boundary conditions.

From the form of the algebra presented in table 1 it is unclear whether a twodimensional non-abelian algebra exists, but after the basis change

$$
\mathrm{L}_{ \pm}=\mathrm{H} \pm \mathrm{B}
$$

one finds two subalgebras spanned by $\left\{\mathrm{L}_{+}, \mathrm{P}\right\}$ and $\left\{\mathrm{L}_{-}, \mathrm{P}\right\}$ respectively. We choose the latter as the subalgebra the generators of which are imposed as constraints (the former leads again to identical conclusions upon redefinition of the radial coordinate). The fixed element $K$ in (5.11) is then required to be of the form $K=\mathrm{L}_{+}+\gamma_{\mathrm{M}} \mathrm{M}$, with $\gamma_{\mathrm{M}}$ arbitrary such that $\left\langle\mathrm{L}_{-}, K\right\rangle$ is non-zero and the constraints associated to $\mathrm{P}$ and $\mathrm{L}_{-}$become second-class. This translates to boundary conditions reminiscent of highest-weight gauge

$$
\begin{aligned}
& A=\left(e^{\rho} \mathrm{L}_{+}+\mathcal{L}(t) e^{-\rho} \mathrm{L}_{-}+\mathcal{T}(t) \mathrm{P}_{1}+\gamma_{\mathrm{M}} \mathrm{M}\right) d t+d \rho \mathrm{P}_{1} \\
& \mathcal{X}=e^{\rho} \bar{X} \mathrm{~L}_{+}+\mathcal{O}(1)
\end{aligned}
$$

where the radial dependence is captured by the group element $b=\exp (\rho \mathrm{P})$, and the form of the dilaton field follows from the universal boundary condition (5.2) where we set $\partial_{t} f=\bar{X}$.

Under gauge transformations with generator $\lambda=\lambda_{+} \mathrm{L}_{+}+\lambda_{-} \mathrm{L}_{-}+\lambda_{\mathrm{P}} \mathrm{P}+\lambda_{\mathrm{M}} \mathrm{M}$ that preserve the form of $A$ in (6.19) the state-dependent functions transform as

$$
\delta \mathcal{T}=\lambda_{+} \mathcal{T}^{\prime}+\lambda_{+}^{\prime} \mathcal{T}-\lambda_{+}^{\prime \prime} \quad \delta \mathcal{L}=\lambda_{+} \mathcal{L}^{\prime}+2 \mathcal{L} \lambda_{+}^{\prime}-\frac{1}{2}\left(\lambda_{\mathrm{M}}^{\prime} \mathcal{T}+\lambda_{\mathrm{M}}^{\prime \prime}\right)
$$

This transformation law is the hallmark of twisted warped symmetry. Both central charge and $\mathfrak{u}(1)$ level are zero so that the only non-trivial cocycle is the twist, $\kappa=1$ in the conventions of $[84,85]$. The fact that the symmetry algebra $i \mathfrak{s o}(1,1)^{c}$ is naturally related to warped Virasoro symmetries was also found in the work [38] that studied three-dimensional Chern-Simons theory based on two copies of $\mathfrak{i s o}(1,1)^{c}$ and uncovered a warped Virasoro symmetry as asymptotic symmetry algebra. We therefore expect the boundary action to be related to some version of the warped Schwarzian action of [85] at these specific values of the central charges. We will find this expectation confirmed below. 
In order to obtain some geometric insight into the boundary condition, we can recover from (6.19) the zweibein

$$
e^{0}=\left(e^{\rho}+\mathcal{M} e^{-\rho}\right) d t \quad e^{1}=\mathcal{T} d t+d \rho
$$

and thus the Carrollian structure

$$
q=(\mathcal{T} d t+d \rho)^{2} \quad n=\frac{1}{e^{\rho}+\mathcal{M} e^{-\rho}}\left(\partial_{t}-\mathcal{T} \partial_{\rho}\right)
$$

where $q$ is the degenerate metric and $n$ denotes the vector field lying in its kernel. This AdSC spacetime can be regarded as a null surface embedded in three-dimensional AdS space. More precisely, starting from Poincaré patch coordinates in three dimensions

$$
d s^{2}=d \rho^{2}-e^{\rho} d x^{+} d x^{-}
$$

the null-surface $x^{-}=0$ has induced degenerate metric and normal vector of the form

$$
q=d \rho^{2} \quad n=e^{-\rho} \partial_{+}
$$

coinciding with (6.22) in the case $\mathcal{T}=\mathcal{M}=0$ which one may regard as the vacuum configurations of (6.19). The boundary of the null-surface is obtained in the limit $\rho \rightarrow \infty$. The boundary coordinate $t$ of the BF theory is subsequently interpreted as a null-coordinate along the boundary of $\mathrm{AdS}_{3}$.

We address one more interesting feature of the AdSC geometry (6.22). When approaching the boundary $\rho \rightarrow \infty$, the degenerate metric $q$ diverges while the vector $n$ goes to zero. But upon introducing the boundary defining function $\Omega=e^{-\rho}$ and rescaling both quantities by the conformal factor $q \rightarrow \Omega^{2} q, n \rightarrow \Omega^{-1} n$, one finds that the pull-back of the AdSC structure to the boundary $\Omega=0$ yields

$$
q=0 \quad n=\partial_{t} .
$$

Since $\Omega$ is defined only up to a non-vanishing factor, one finds as the boundary structure of AdSC spacetimes a conformal class of vectors together with the zero metric. This is precisely the same boundary structure as $2 \mathrm{~d}$ asymptotically flat spacetimes. So not only share these geometries the same local symmetry group, i.e., the Poincaré group, but also the same conformal boundary structure.

We turn now to the boundary action that can be obtained from the above boundary conditions. Parametrizing the group element as

$$
g=e^{y \mathrm{~L}_{+}} e^{\phi_{-} \mathrm{L}_{-}} e^{\phi_{\mathrm{P}} \mathrm{P}} e^{z \mathrm{Z}}
$$

the current constraints implied by (6.19)

$$
\left.g^{-1} \partial_{t} g\right|_{+}=\left.1 \quad g^{-1} \partial_{t} g\right|_{\mathrm{M}}=\gamma_{\mathrm{M}}
$$

allow to solve $\phi_{-}, \phi_{\mathrm{P}}$ algebraically in terms of $y, z$ due to the second-class nature of the constraints. Plugging the solutions into the action (6.9) leads to

$$
I=\bar{X} \int d t\left(z^{\prime}\left(\log y^{\prime}\right)^{\prime}-z^{\prime \prime}+\gamma_{\mathrm{M}}\left(\log y^{\prime}\right)^{\prime}\right) .
$$


We recognize this as a form of the twisted warped action of $[85,86] \cdot{ }^{10}$ In the parametrization (6.26), the two free functions in the connection (6.19) are given by

$$
\mathcal{T}=-\left(\log y^{\prime}\right)^{\prime} \quad \mathcal{L}=\frac{1}{2}\left(z^{\prime}\left(\log y^{\prime}\right)^{\prime}-z^{\prime \prime}-\gamma_{\mathrm{M}}\left(\log y^{\prime}\right)^{\prime}\right)
$$

and the equations of motion are equivalent to their conservation

$$
\partial_{t} \mathcal{T}=\partial_{t} \mathcal{L}=0
$$

Finally, this action is invariant under the finite transformations

$$
y \mapsto a+y c \quad z \mapsto z+d+b y
$$

that correspond to the global symmetry group of $\operatorname{AdSC}$, i.e., $\operatorname{ISO}(1,1)^{c}$. As in the Schwarzian case discussed above, these transformations should be interpreted as gauge transformation arising due to the redundancy in the reduction.

As a final comment we remind the reader that the AdSC theory discussed here exhibits the same algebra as dS-Galilei and 2d Minkowski space. Consequently, the boundary conditions (6.19) are a priori also applicable to those theories albeit with different geometrical interpretation of generators and free fields. Indeed, the work [86] found essentially the same action, but with complex fields, as boundary action in the case of (Euclidean) flat space and found an explicit relation to a scaling limit of the effective action of the complex SYK model.

\subsection{Other kinematical algebras}

In the last two sections we constructed boundary actions for hyperbolic space/Euclidean $\mathrm{AdS}_{2}$ and AdS-Carroll 2 as representatives for all kinematical spacetimes based on $\mathfrak{s o}(2,1)$ and $i \mathfrak{s o}(1,1)^{c}$. Disregarding the sphere and the static spacetime it remains to discuss spacetimes with symmetry algebras iso $(2)^{c}$ and Heisenberg ${ }^{c c}$, i.e., spacetimes in the second and fourth block of table 1 , in order to cover all kinematical algebras with invariant metric.

According to the algorithm of section 5.2 we should look for even-dimensional noncommutative subalgebras that we can use to write down boundary conditions that reduce the dynamics of the point particle action (5.7). Starting with the doubly extended Heisenberg algebra that corresponds to e.g., flat Carroll spacetime, we find that the form of the algebra does not allow for any such subalgebra. The algebra is nilpotent from which follows that all two-dimensional subalgebras are abelian. Furthermore, the center of the algebra is two-dimensional so that any four-dimensional subalgebra necessarily contains an element that commutes with all of its remaining generators. Boundary conditions that derive from the algebraic structure and act by restricting the charges $Q$ (5.9) will therefore lead to first-class constraints and gauge symmetry in the boundary action.

In the case of the algebra $\mathfrak{i s o}(2)^{c}$ corresponding to e.g., AdS-Galilei, it is straightforward to show that no two-dimensional non-abelian subalgebra exists unless one allows the algebra, and thus the fields, to become complex.

\footnotetext{
${ }^{10}$ Upon redefining $y=e^{i u}$ this result agrees with the zero-temperature version of [41].
} 
This means that the procedure in section 5 needs to be modified to construct boundary actions for these examples. The simplest 'modification' is to not enforce any constraints at all, i.e., to stick with the most general boundary conditions. In this case the boundary action is always given by (5.7).

We leave the construction of alternative boundary actions for these cases for future work.

\section{Applications and generalizations}

The main conclusion of our work is that it is done - in particular, we have provided answers to the questions posed in the introduction: there is a $\mathrm{NC}$ version of $2 \mathrm{~d}$ dilaton gravity, discussed in section 3.4; the Schwarzian analogue for the AdS-Carroll 2 limit of JT gravity is the twisted warped action, discussed in section 6.2.

Rather than summarizing our results in past tense, we address potential applications of various limits of JT gravity and their dilaton generalizations in section 7.1, and point out some interesting and viable generalizations in section 7.2.

\subsection{Selected applications}

Without claiming to be complete, here is a list of selected applications of our results, listed by order of appearance:

- Light cone theory. The light cone Lagrangian (3.8) was easy enough to construct, but the physical interpretation of the latter remains to be explored. As advertised in [39] the light cone theory might be interesting in relation to asymptotically flat spacetimes in three dimensions; cf. [83] for the four-dimensional case. Thus, likely applications of (3.8) are in the context of three-dimensional asymptotically flat gravity. Moreover, it could be interesting to study a dilaton gravity-inspired generalization of the light cone theory, analogous to (3.5).

- Newton-Cartan dilaton gravity. The NC dilaton gravity action (3.21) has the same status for NC gravity as generic dilaton gravity (3.5) (or its torsionful generalization below that equation) for Riemann-Cartan gravity. While we have shown numerous similarities - the existence of constant and linear dilaton sectors, the exact solubility of the equations of motion, the possibility to accommodate higherdimensional gravity models - we have provided only one example. Given the vast literature of ordinary $2 \mathrm{~d}$ dilaton gravity (see the table in [58] for a selected list of models) it seems likely that there are several interesting NC dilaton gravity models waiting to be applied.

- Carroll dilaton gravity. We were slightly less explicit concerning Carroll dilaton gravity (3.33), but clearly the same techniques that we used to study NC dilaton gravity can be applied there. Similar remarks concerning applications as in the previous item apply to this case, with the additional interesting option to explore the relationship to other field theories on null manifolds, see, e.g., [87, 88]. 
- The rest. We were even less explicit regarding several other entries in table 1, largely because we do not have a good proposal for applications of, say, the para-Galilei or the static case. Nevertheless, such applications may exist, and if they do, again the same techniques as for $\mathrm{NC}$ dilaton gravity can be applied to construct and solve JT-like and dilaton gravity-like models that build upon these homogeneous spaces.

- Boundary actions. The loosest set of boundary conditions for any BF-type model always leads to a boundary action describing a particle on a group manifold (5.7). Since for many gravity-inspired applications something like Brown-Henneaux boundary conditions is preferred, it will be rewarding to apply the Hamiltonian reduction of the boundary action described in section 5.2 to other models of interest. Such applications will be analogous to the example of AdS-Carroll 2 we provided in section 6.2, which led to a twisted warped action (6.28).

- Lifshitz, Schrödinger et al. The theories mentioned in appendices B, C also lead to BF theories, and thus there will be 'dilaton gravity' versions thereof, analogous to section 3.4. Some of these models may be useful for applications in the context of Lifshitz or Schrödinger holography.

Besides the rather direct applications above there are also several exciting potential applications that will require - in some cases substantial - further input (here we order the items by likelihood of substantial progress):

- JT/SYK-like correspondences. Possibly the largest set of potential applications is to generalize the JT/SYK correspondence to interesting limiting cases. The fact that our models emerge from a limit of JT suggests that one can also implement a similar limit on the dual quantum mechanics model. A concrete example realizing this expectation is the flat space/cSYK correspondence discussed in [86]. Of course, taking limits is just the first step in a much bigger picture. Many of the developments in the JT/SYK correspondence associated with chaos, relation to random matrix models, applications to quantum gravity, etc. (see [13-15] for reviews) could be transposed to these limiting cases. We are convinced that this route can lead to numerous exciting discoveries in the near future.

- Relation to three-dimensional models. There is a long-term relationship between three- and two-dimensional gravity, since the latter arises by dimensional reduction of the former. But the relation is deeper than that. Also at a technical level there are numerous similarities, particular in the respective gauge theoretic formulations as Chern-Simons and BF-theories - both are topological gauge theories of Schwarz type and can feature non-trivial edge modes, depending on the boundary conditions. For metric BF theories the relation should be straightforward, since the operations of holographic reduction of three-dimensional Chern-Simons to 2d WZW at the boundary commutes with dimensional reduction and yields a corresponding holographic reduction of $2 \mathrm{~d} \mathrm{BF}$ to a corresponding boundary theory, see, e.g., [13]. Indeed many of the findings and tools used for $2+1$ dimensional Chern-Simons, like, 
e.g., in [89-92], can be applied to BF theories. However, for WZW models without invariant metric the situation is not well understood in general; for example WZW models based on Lie algebras admit a Sugawara construction only when possessing an invariant metric [93, 94]. As rôle model we point here to a recent example by Chaturvedi, Papadimitriou, Song and Yu [95] (see also refs. therein), who discussed the dimensional reduction of $\mathrm{AdS}_{3}$ Einstein gravity with Compére-Song-Strominger boundary conditions [73], both on the gravity side and the SYK-side. Analogous applications and relations to higher-dimensional limiting theories should exist for the various limits of JT discussed in our work.

- Thermodynamics and Cardyology. Theories without light cone may have a hard time of defining black hole-like entities. Nevertheless, some of these theories do feature highly entropic objects corresponding to finite temperature states, see e.g. [40, 96], and regardless of their geometric interpretation it is of interest to understand their entropy and, whenever possible, provide a Cardy-inspired microstate counting. A first step in this direction could be a thermodynamical analysis starting with the free energy derived from the on-shell action, analogous to $2 \mathrm{~d}$ dilaton gravity [8].

- Quantization and holography. Quantization of 2d NC dilaton gravity seems a feasible endeavor, due to the quantum integrability of BF-theories (see the review [45]), and might provide an interesting avenue to Galilean and Carrollian quantum gravity and holography. While this leads to various puzzles, like is there something like an information loss problem in any of these theories?, we emphasize that quantizing all our models is possible and could lead to unexpected insights.

- Minkowski and AdS-Carroll. As homogeneous spaces Minkowski and AdSCarroll are both based on the Poincaré algebra and are connected via an exchange of boosts and spatial translations, a relationship that is true in any dimension [49]. The findings of section 6.2 suggest that there might be a deeper relationship that remains to be explored. Two observations triggered our interest: 1.) Since the cosmological constant, that gives AdS its 'boxlike' properties, is still nonzero for AdS-Carroll it might inherit similar advantageous holographic features. 2.) Recent works on celestial amplitudes (for a review see [97]) are based on boost eigenstates, rather than the more conventional momentum eigenstates. This therefore mirrors the above described exchange.

- Relation to $\boldsymbol{J T}$ deformations of $\mathbf{J T}$ ? The limits of JT gravity that we considered break/deform Lorentz symmetries and typically introduce an extra $\mathfrak{u}(1)$. Also Guica's $J T$ deformations [98] break/deform Lorentz symmetries and require an extra $\mathfrak{u}(1)$. Given that the more standard $T \bar{T}$-deformations upon dimensional reduction have a nice interpretation in terms of flow equations in 2d dilaton gravity [99-101], we speculate that a similar relationship could hold between $J T$ deformations and certain limits of JT gravity. Addressing this last point would require a better understanding of the second and third item in this list. 
Most likely there are further potential applications that are missing in our lists above, but let us move on to our final point, generalizations.

\subsection{Selected generalizations}

Here is a list of five classes of generalizations that we consider promising and viable:

- More general boundary conditions. The algorithm explained in section 5.2 imposed a number of assumptions. In particular, $K$ and $\gamma$ in the constraint (5.11) were assumed to be state-independent, and the constraint algebra (5.12) was enforced to be second class. Neither of these assumptions is mandatory, so by relaxing either of them it is possible to construct a whole menagerie of new boundary conditions. A full classification of all possibilities could be worthwhile, for novel applications and for purely theoretical reasons, to get a better understanding of the landscape of boundary conditions.

- Additional topological fields. Our focus was on gravity variables, zweibein and connection, but for some applications it can be of interest to add non-abelian gauge fields. Such a generalization is straightforward in the BF-formulation and will lead to new conserved charges and additional boundary degrees of freedom, but will maintain the topological nature of the theory.

- Supersymmetry and/or higher spins. A variant of the previous item is to include supersymmetry and consider various limits of $2 \mathrm{~d}$ supergravity theories and their boundary actions (see [102] and refs. therein). Similarly, one can extend to higher spin gravity (along the lines of $[68,103])$ and take various limits analogous to the present work. In all these generalizations one would still keep the topological nature of the underlying theory.

- Non-linear gauge symmetries. Chern-Simons models in 3d are rigid, i.e., their most general consistent deformation (in the sense of Barnich and Henneaux [104]) is another Chern-Simons model with the same number of gauge symmetries. A similar story applies to $2 \mathrm{~d}$ BF theories, but with an interesting extension: the most general consistent deformation of $2 \mathrm{~d} \mathrm{BF}$ is a non-linear gauge theory [105] known as Poissonsigma model (PSM) [106]. The Poisson-sigma model Lagrangian

$$
\mathcal{L}_{\mathrm{PSM}}\left[X^{I}, A_{I}\right]=X^{I} d A_{I}+\frac{1}{2} P^{I J}\left(X^{K}\right) A_{I} \wedge A_{J}
$$

features again scalars $X^{I}$ and connection 1-forms $A_{I}$ and is first order in derivatives, just like BF theories, but the interpretation of the $X^{I}$ is now as target space coordinates spanning a Poisson manifold. The main new ingredient is an arbitrary Poisson-tensor $P^{I J}=-P^{J I}$, subject to the non-linear Jacobi identities $P^{I L} \partial_{L} P^{J K}+\operatorname{cycl}(I, J, K)=0$. In this languages, BF theories (with or without metric) are merely special cases of PSMs with linear Poisson tensor, and the limits we have taken can be rephrased as corresponding limits of the Poisson tensor. Secretly, we have already introduced specific PSMs when providing the action for $2 \mathrm{~d}$ 
dilaton gravity (3.5), NC dilaton gravity (3.21) [see particularly the non-linear gauge symmetries (3.26)] and Carroll dilaton gravity (3.33). In order to construct boundary actions for all these models it will be necessary to understand how this can be done in generic PSMs, which is why this particular generalization seems eminently worthwhile.

- Adding matter. Topological gauge theories like BF or PSM have numerous technical advantages, but miss an important aspect of physics, namely locally propagating physical degrees of freedom. A possible compromise between the conflicting desires of keeping the model simple and physically rich is to couple BF theories (or PSMs) to matter degrees of freedom. Some consequences of such a coupling are reviewed in the context of $2 \mathrm{~d}$ dilaton gravity in [30]. While ambitious, it could pay off to add matter to theories like NC or Carroll dilaton gravity and address some questions concerning 1-loop effects, backreactions, etc.

While further generalizations not envisaged here are conceivable, the lists above provide already a plethora of possibilities for future research.

\section{Acknowledgments}

We thank Arash Ranjbar, Dieter Van den Bleeken and José Figueroa-O'Farrill for useful discussions. We want to thank José Figueroa-O'Farrill for valuable advice concerning metric Lie algebras.

DG was supported by the Austrian Science Fund (FWF), projects P 30822-N27 and P 32581-N27. During the start of this project the research of SP was supported by the ERC Advanced Grant "High-Spin-Grav" and by FNRS-Belgium (Convention FRFC PDR T.1025.14 and Convention IISN 4.4503.15). SP was supported by the Leverhulme Trust Research Project Grant (RPG-2019-218) "What is Non-Relativistic Quantum Gravity and is it Holographic?". The work of JH is supported by the Royal Society University Research Fellowship "Non-Lorentzian Geometry in Holography" (grant number UF160197). JS was supported by the Erwin-Schrödinger fellowship J-4135 of the Austrian Science Fund (FWF) and by the NSF grant 1707938.

\section{A Metric Lie algebras of low dimension}

We summarize low-dimensional real Lie algebras that admit an invariant metric (see, e.g., [107] for a review). The Roman numerals refer to the name of specific Lie algebras according to Bianchi's classification [64, 65], cf., our summary table 1.

$\operatorname{dim}(\mathfrak{g})=\mathbf{1}$ and $\operatorname{dim}(\mathfrak{g})=\mathbf{2}$. For one and two dimensional Lie algebras solely the abelian Lie algebras admit an invariant metric.

$\operatorname{dim}(\mathfrak{g})=3$. Three dimensional Lie algebras that admit an invariant metric are either abelian (denoted by Bianchi as I) or simple and amount to three distinct cases in total. The simple one is either $\mathfrak{s l}(2, \mathbb{R}) \simeq \mathfrak{s o}(2,1) \simeq \mathfrak{s o}(1,2) \simeq \mathfrak{s u}(1,1) \simeq$ VII, which correspond 
to the $(\mathrm{A}) \mathrm{dS}$ and light cone cases or $\mathfrak{s o}(3) \simeq \mathfrak{s u}(2) \simeq$ IX leading to the sphere (or Euclidean de Sitter), see table 1.

$\operatorname{dim}(\mathfrak{g})=4$. There exist five metric Lie algebras of dimension four. One of them is the abelian Lie algebra. Additionally, there are the trivial central extensions of the two simple three dimensional cases $\mathbb{R} \times \mathfrak{s l}(2, \mathbb{R})$ and $\mathbb{R} \times \mathfrak{s o}(3, \mathbb{R})$. They serve in this work as a starting point for contractions to the remaining two metric solvable algebras given by the (centrally) extended Euclidean $\mathfrak{i s o}(2)^{c}=\mathrm{VII}_{0}^{c}$ or Poincaré algebra iso $(1,1)^{c}=\mathrm{VI}_{0}^{c}$ (which are isomorphic to their (A)dS-Carrollian and (A)dS-Galilean cousins, cf., table 1). The centrally extended Poincaré algebra is also known as Maxwell or Boidol algebra and the Euclidean case is sometimes referred to as oscillator algebra.

$\operatorname{dim}(\mathfrak{g})=\mathbf{5}$. For five dimensional Lie algebras there are six Lie algebras that admit an invariant metric, five of which are trivial central extensions of the four dimensional ones. The remaining unique indecomposable metric Lie algebra is Heisenberg ${ }^{c c} \simeq \mathrm{II}^{c c}$ of our table 1 and underlies the doubly extended Carrollian and Galilean theories.

$\operatorname{dim}(\mathfrak{g})>5$. Beyond dimension five one can use the classifications of metric Lie algebras that have been obtained for signature $(n, 1)$ [108] (see also section 4 in [109]), $(n, 2)$ [110] and $(n, 3)$ [111]. Kinematical Lie algebras and their relation to metric Lie algebras in any dimension have also been studied in [39]. The higher-dimensional algebras do not play any rôle in the present work.

\section{B Lifshitz, Schrödinger and 1/c expanded BF theories}

Here we provide all the necessary data to construct Lifshitz, Schrödinger and $1 / c$ expanded $\mathrm{BF}$ theories. None of them (even with central extensions taken into account) lead to metric BF theories.

The Lifshitz algebra is spanned by dilatations (D), time (H) and space (P) translations

$$
[\mathrm{D}, \mathrm{H}]=z \mathrm{H} \quad[\mathrm{D}, \mathrm{P}]=\mathrm{P}
$$

with the most general (degenerate) invariant bilinear form given by

$$
\langle\mathrm{D}, \mathrm{D}\rangle=\chi
$$

This algebra has no nontrivial central extensions.

For $z=2$ this algebra can be enhanced to the Schrödinger algebra by adding mass $M$ and special conformal transformations $\mathrm{C}$,

$$
\begin{aligned}
& {[\mathrm{B}, \mathrm{P}]=\mathrm{M}} \\
& {[\mathrm{D}, \mathrm{H}]=2 \mathrm{H}} \\
& {[\mathrm{D}, \mathrm{P}]=\mathrm{P}} \\
& {[\mathrm{D}, \mathrm{B}]=-\mathrm{B}} \\
& {[\mathrm{C}, \mathrm{P}]=\mathrm{B}} \\
& {[\mathrm{C}, \mathrm{D}]=2 \mathrm{C} \text {. }}
\end{aligned}
$$

This algebra has the same bilinear form as the Lifshitz case, but admits one central extension $[\mathrm{H}, \mathrm{C}]=\mathrm{Z}$. Even including this central extension leaves the bilinear form degenerate. 
The nonzero commutation relations of the $1 / c$ expanded Poincaré algebra (till level 1 ) are given by [112]

$$
[B, H]=P \quad[B, P]=H^{(1)} \quad\left[B, H^{(1)}\right]=P^{(1)} \quad\left[B^{(1)}, H\right]=P^{(1)}
$$

with the most general degenerate invariant bilinear form

$$
\begin{aligned}
& \langle\mathrm{B}, \mathrm{B}\rangle=\chi_{\mathrm{B}} \quad\langle\mathrm{B}, \mathrm{H}\rangle=\chi_{\mathrm{BH}} \quad\langle\mathrm{H}, \mathrm{H}\rangle=\chi_{\mathrm{H}} \\
& \left\langle\mathrm{B}, \mathrm{B}^{(1)}\right\rangle=\chi_{\mathrm{BB}^{(1)}} \quad\left\langle\mathrm{H}, \mathrm{B}^{(1)}\right\rangle=\chi_{\mathrm{HB}^{(1)}} \quad\left\langle\mathrm{B}^{(1)}, \mathrm{B}^{(1)}\right\rangle=\chi_{\mathrm{B}^{(1)}} \text {. }
\end{aligned}
$$

These algebras appear in the context of Lie algebra expansions (see, e.g., [113-115]) which have recently found applications in the context of general relativity. The notation can be explained as follows. Take the Cartan connection 1-form, say

$$
A=\mathrm{B} \omega+\tau \mathrm{H}+e \mathrm{P}+\cdots
$$

where the dots denote possibly other elements in the Lie algebra. When the 1-forms $\tau, e$ etc. depend on the speed of light (as is the case for the Poincare algebra) we can assume that the $c$ dependence is analytic so that we can Taylor expand

$$
\tau=\sum_{n=0}^{\infty} \tau_{(n)} c^{-2 n}
$$

where we assume only even powers of $c^{-1}$. Substituting this expansion into the Cartan connection and defining a Lie algebra generator for each $\tau_{(n)}$ we end up with the Lie algebra elements $\mathrm{H}^{(n)}=\mathrm{H}^{-2 n}$ (and analog for the remaining generators). Since the structure constants can also depend on $c^{-2}$ it is possible that the bracket of a level $n$ with a level $m$ generator gives a generator of a level that is strictly larger than $m+n$. The terms with superscript (1) denote generators of next to leading order ('level 1'). The level 0 algebra, where one quotients out all Lie algebra elements of level bigger than 0 is again the Galilei algebra. The algebra (B.4) is the algebra where all levels $n>1$ have been modded out.

The $1 / c$ expanded algebra in $1+1$ dimensions allows for 6 nontrivial central extensions $\mathrm{M}, \mathrm{Z}_{1}$ to $\mathrm{Z}_{5}$

$$
\begin{aligned}
& {[\mathrm{B}, \mathrm{H}]=\mathrm{P} \quad[\mathrm{B}, \mathrm{P}]=\mathrm{H}^{(1)} \quad\left[\mathrm{B}, \mathrm{H}^{(1)}\right]=\mathrm{P}^{(1)}+\mathrm{Z}_{1} \quad\left[\mathrm{~B}^{(1)}, \mathrm{H}\right]=\mathrm{P}^{(1)}+\mathrm{Z}_{2}} \\
& {[\mathrm{H}, \mathrm{P}]=\mathrm{M} \quad\left[\mathrm{H}, \mathrm{P}^{(1)}\right]=\mathrm{Z}_{3} \quad\left[\mathrm{P}, \mathrm{H}^{(1)}\right]=-\mathrm{Z}_{3} \quad\left[\mathrm{~B}, \mathrm{~B}^{(1)}\right]=\mathrm{Z}_{4}} \\
& {\left[\mathrm{~B}, \mathrm{P}^{(1)}\right]=\mathrm{Z}_{6} \quad\left[\mathrm{P}, \mathrm{B}^{(1)}\right]=-\mathrm{Z}_{6} \text {. }}
\end{aligned}
$$

A necessary condition for a non-degenerate invariant metric would be to only add two central extensions to (B.4), cf., (4.3). We have checked that the addition of M and any of the other central extensions does not lead to such a non-degenerate invariant metric.

\section{Coadjoint theories and their limits}

For completeness we briefly mention another class of theories where the invariant metric stays, basically by construction, non-degenerate under limits. These theories have the distinctive feature that the algebraic structure generalizes to generic dimension (see, e.g., [39]). 
They are based on so called coadjoint Lie algebras, which are a subcase of the already mentioned double extension [46]. Given a Lie algebra $\mathfrak{g}$ they are defined on the vector space direct sum $\mathfrak{g} \dot{+} \mathfrak{g}^{*}$ (spanned by $\mathrm{e}_{\alpha}$ and $\mathrm{e}^{* \alpha}$, respectively) by the commutation relations

$$
\left[\mathbf{e}_{\alpha}, \mathbf{e}_{\beta}\right]=c_{\alpha \beta}{ }^{\gamma} \mathrm{e}_{\gamma} \quad\left[\mathrm{e}_{\alpha}, \mathrm{e}^{* \beta}\right]=-c_{\alpha \gamma}{ }^{\beta} \mathrm{e}^{* \gamma} \quad\left[\mathrm{e}^{* \alpha}, \mathrm{e}^{* \beta}\right]=0
$$

and the invariant metric

$$
\left\langle\mathrm{e}_{\alpha}, \mathrm{e}_{\beta}\right\rangle=h_{\alpha \beta} \quad\left\langle\mathrm{e}_{\alpha}, \mathrm{e}^{* \beta}\right\rangle=\delta_{\alpha}^{\beta}
$$

which is non-degenerate due to the second term. The other part of the invariant metric $h_{\alpha \beta}$ is some arbitrary (possibly degenerate or zero) invariant symmetric bilinear form on $\mathfrak{g}$. The extension of $\mathfrak{g}$ by $\mathfrak{g}^{*}$ is not central in general.

We discuss all limits and theories at once. Starting with (A)dS this means that additionally to commutation relations (4.1) we have the nonzero commutators

$$
\begin{aligned}
{\left[\mathrm{B}, \mathrm{H}^{*}\right] } & =-\hat{c}^{2} \mathrm{P}^{*} & {\left[\mathrm{~B}, \mathrm{P}^{*}\right] } & = \pm \hat{C}^{2} \mathrm{H}^{*} \\
{\left[\mathrm{H}, \mathrm{B}^{*}\right] } & =\hat{\Lambda} \mathrm{P}^{*} & {\left[\mathrm{H}, \mathrm{P}^{*}\right] } & =\mp \hat{C}^{2} \mathrm{~B}^{*} \\
{\left[\mathrm{P}, \mathrm{B}^{*}\right] } & =-\hat{\Lambda} \mathrm{H}^{*} & {\left[\mathrm{P}, \mathrm{H}^{*}\right] } & =\hat{c}^{2} \mathrm{~B}^{*}
\end{aligned}
$$

and in addition to the invariant metric (4.2), which corresponds to the $h_{\alpha \beta}$ part described above, we have by construction

$$
\left\langle\mathrm{B}, \mathrm{B}^{*}\right\rangle=1 \quad\left\langle\mathrm{H}, \mathrm{H}^{*}\right\rangle=1 \quad\left\langle\mathrm{P}, \mathrm{P}^{*}\right\rangle=1 .
$$

As already discussed, the part of the invariant metric given by (4.2) is degenerate under limits; it is (C.4) that guarantees the existence of the non-degenerate invariant metric. The limit of the algebra $\mathfrak{g}$ induces the limits of $\mathfrak{g}^{*}$, which are well defined by construction (a fact that generalizes under certain circumstances to double extensions [116]).

Having provided the Lie algebra and the invariant metric it is now an easy exercise to write down the action by just inserting into the Lagrangian (2.4). There are two curious features of the BF theories based on coadjoint Lie algebras. First, the existence of the algebras and their invariant metric is not constrained to $1+1$ dimensions and as such these are the algebras that are interesting candidates for generalization to higher dimension (see section 7 of [39] for details). Second, a comment in relation to $2+1$ dimensions and ChernSimons theories: the coadjoint Lie algebras are precisely the ones that can be written as $2+1$ dimensional BF theories, see, e.g., section 6.2.2. [45]. In particular, the threedimensional Poincaré algebra can be regarded as the coadjoint algebra of $\mathfrak{s o}(3)$ so that its Chern-Simons theory can be equivalently regarded as a $(2+1)$-dimensional BF theory of $\mathfrak{s o}(3)$. For further recent works based on these algebras we refer to [117-119].

\section{Matrix representations}

We collect in this appendix matrix representations that are useful in the calculations of section 5 . In the following, let $X_{i j}^{(n)}$ denote the $n \times n$ matrix with entry 1 in the slot $i j$ and zero everywhere else. 
The $\mathfrak{s l}(2, \mathbb{R})$ basis $\mathrm{L}_{+}, \mathrm{L}_{-}, \mathrm{L}_{0}$ with commutation relations

$$
\left[\mathrm{L}_{m}, \mathrm{~L}_{n}\right]=(m-n) \mathrm{L}_{m+n} \quad m, n=-1,0,1
$$

used in the calculation of section 6.1 is conveniently represented as

$$
\mathrm{L}_{+}=X_{21}^{(2)} \quad \mathrm{L}_{-}=-X_{12}^{(2)} \quad \mathrm{L}_{0}=\frac{1}{2}\left(X_{11}^{(2)}-X_{22}^{(2)}\right)
$$

The invariant bilinear in this basis is given by

$$
\left\langle\mathrm{L}_{+}, \mathrm{L}_{-}\right\rangle=2 \quad\left\langle\mathrm{~L}_{0}, \mathrm{~L}_{0}\right\rangle=-1 .
$$

The AdSC algebra in the basis $\mathrm{L}_{+}, \mathrm{L}_{-}, \mathrm{P}, \mathrm{M}$ used in section 6.2 with commutation relations

$$
\left[\mathrm{L}_{ \pm}, \mathrm{P}\right]= \pm \mathrm{L}_{ \pm} \quad\left[\mathrm{L}_{+}, \mathrm{L}_{-}\right]=-2 \mathrm{M}
$$

can be represented by

$$
\mathrm{L}_{+}=X_{12}^{(3)} \quad \mathrm{L}_{-}=X_{23}^{(3)} \quad \mathrm{P}=X_{22}^{(3)} \quad \mathrm{M}_{+}=-2 X_{13}^{(3)} .
$$

The invariant bilinear form in this basis is given by

$$
\left\langle\mathrm{L}_{+}, \mathrm{L}_{-}\right\rangle=2 \quad\langle\mathrm{P}, \mathrm{M}\rangle=1 .
$$

Finally, the doubly extended Heisenberg algebra has the matrix representation

$$
\mathrm{B}=X_{12}^{(5)}-X_{35}^{(5)} \quad \mathrm{P}=X_{13}^{(5)}+X_{25}^{(5)} \quad \mathrm{Z}=-X_{14}^{(5)} \quad \mathrm{M}=2 X_{15}^{(5)} \quad \mathrm{H}=X_{23}^{(5)}+X_{34}^{(5)} .
$$

Open Access. This article is distributed under the terms of the Creative Commons Attribution License (CC-BY 4.0), which permits any use, distribution and reproduction in any medium, provided the original author(s) and source are credited.

\section{References}

[1] B.M. Barbashov, V.V. Nesterenko and A.M. Chervyakov, The solitons in some geometrical field theories, Theor. Math. Phys. 40 (1979) 572 [INSPIRE].

[2] E. D'Hoker and R. Jackiw, Liouville Field Theory, Phys. Rev. D 26 (1982) 3517 [InSPIRE].

[3] C. Teitelboim, Gravitation and Hamiltonian Structure in Two Space-Time Dimensions, Phys. Lett. B 126 (1983) 41 [InSPIRE].

[4] R. Jackiw, Lower Dimensional Gravity, Nucl. Phys. B 252 (1985) 343 [INSPIRE].

[5] R.B. Mann, A. Shiekh and L. Tarasov, Classical and Quantum Properties of Two-dimensional Black Holes, Nucl. Phys. B 341 (1990) 134 [InSPIRE].

[6] M. Cadoni and S. Mignemi, Entropy of $2-D$ black holes from counting microstates, Phys. Rev. D 59 (1999) 081501 [hep-th/9810251] [INSPIRE].

[7] S. Cacciatori, A.H. Chamseddine, D. Klemm, L. Martucci, W.A. Sabra and D. Zanon, Noncommutative gravity in two dimensions, Class. Quant. Grav. 19 (2002) 4029 [hep-th/0203038] [INSPIRE]. 
[8] D. Grumiller and R. McNees, Thermodynamics of black holes in two (and higher) dimensions, JHEP 04 (2007) 074 [hep-th/0703230] [INSPIRE].

[9] A. Sen, Entropy Function and $A d S_{2} / C F T_{1}$ Correspondence, JHEP 11 (2008) 075 [arXiv:0805.0095] [INSPIRE].

[10] T. Hartman and A. Strominger, Central Charge for AdS 2 Quantum Gravity, JHEP 04 (2009) 026 [arXiv: 0803.3621] [INSPIRE].

[11] A. Castro, D. Grumiller, F. Larsen and R. McNees, Holographic Description of AdS $S_{2}$ Black Holes, JHEP 11 (2008) 052 [arXiv:0809.4264] [INSPIRE].

[12] J. Maldacena, D. Stanford and Z. Yang, Conformal symmetry and its breaking in two dimensional Nearly Anti-de-Sitter space, PTEP 2016 (2016) 12C104 [arXiv:1606.01857] [INSPIRE].

[13] T.G. Mertens, The Schwarzian theory — origins, JHEP 05 (2018) 036 [arXiv: 1801.09605] [INSPIRE].

[14] G. Sárosi, $A d S_{2}$ holography and the SYK model, PoS(Modave2017) (2018) 001 [arXiv: 1711.08482] [INSPIRE].

[15] Y. Gu, A. Kitaev, S. Sachdev and G. Tarnopolsky, Notes on the complex Sachdev-Ye-Kitaev model, JHEP 02 (2020) 157 [arXiv:1910.14099] [INSPIRE].

[16] J.S. Cotler et al., Black Holes and Random Matrices, JHEP 05 (2017) 118 [Erratum ibid. 09 (2018) 002] [arXiv: 1611.04650] [InSPIRE].

[17] P. Saad, S.H. Shenker and D. Stanford, JT gravity as a matrix integral, arXiv:1903.11115 [INSPIRE].

[18] S. Dubovsky, V. Gorbenko and M. Mirbabayi, Asymptotic fragility, near AdS 2 holography and TT, JHEP 09 (2017) 136 [arXiv:1706.06604] [INSPIRE].

[19] J. Cardy, The TT deformation of quantum field theory as random geometry, JHEP 10 (2018) 186 [arXiv: 1801.06895] [InSPIRE].

[20] J. Maldacena and X.-L. Qi, Eternal traversable wormhole, arXiv:1804.00491 [INSPIRE].

[21] K. Goto, H. Marrochio, R.C. Myers, L. Queimada and B. Yoshida, Holographic Complexity Equals Which Action?, JHEP 02 (2019) 160 [arXiv:1901.00014] [INSPIRE].

[22] D. Harlow and D. Jafferis, The Factorization Problem in Jackiw-Teitelboim Gravity, JHEP 02 (2020) 177 [arXiv: 1804.01081] [INSPIRE].

[23] A. Kitaev and S.J. Suh, Statistical mechanics of a two-dimensional black hole, JHEP 05 (2019) 198 [arXiv: 1808.07032] [InSPIRE].

[24] G. Penington, Entanglement Wedge Reconstruction and the Information Paradox, JHEP 09 (2020) 002 [arXiv: 1905.08255] [INSPIRE].

[25] A. Almheiri, N. Engelhardt, D. Marolf and H. Maxfield, The entropy of bulk quantum fields and the entanglement wedge of an evaporating black hole, JHEP 12 (2019) 063 [arXiv: 1905. 08762] [INSPIRE].

[26] A. Almheiri, R. Mahajan, J. Maldacena and Y. Zhao, The Page curve of Hawking radiation from semiclassical geometry, JHEP 03 (2020) 149 [arXiv:1908.10996] [INSPIRE].

[27] A. Almheiri, R. Mahajan and J. Maldacena, Islands outside the horizon, arXiv:1910.11077 [INSPIRE]. 
[28] A. Almheiri, T. Hartman, J. Maldacena, E. Shaghoulian and A. Tajdini, Replica Wormholes and the Entropy of Hawking Radiation, JHEP 05 (2020) 013 [arXiv:1911.12333] [INSPIRE].

[29] G. Penington, S.H. Shenker, D. Stanford and Z. Yang, Replica wormholes and the black hole interior, arXiv:1911.11977 [INSPIRE].

[30] D. Grumiller, W. Kummer and D.V. Vassilevich, Dilaton gravity in two-dimensions, Phys. Rept. 369 (2002) 327 [hep-th/0204253] [INSPIRE].

[31] C.G. Callan, Jr., S.B. Giddings, J.A. Harvey and A. Strominger, Evanescent black holes, Phys. Rev. D 45 (1992) 1005 [hep-th/9111056] [INSPIRE].

[32] A. Achucarro and P.K. Townsend, A Chern-Simons Action for Three-Dimensional anti-de Sitter Supergravity Theories, Phys. Lett. B 180 (1986) 89 [INSPIRE].

[33] E. Witten, (2+1)-Dimensional Gravity as an Exactly Soluble System, Nucl. Phys. B 311 (1988) 46 [INSPIRE].

[34] G. Papageorgiou and B.J. Schroers, A Chern-Simons approach to Galilean quantum gravity in $2+1$ dimensions, JHEP 11 (2009) 009 [arXiv:0907.2880] [INSPIRE].

[35] G. Papageorgiou and B.J. Schroers, Galilean quantum gravity with cosmological constant and the extended q-Heisenberg algebra, JHEP 11 (2010) 020 [arXiv:1008.0279] [INSPIRE].

[36] J. Hartong, Y. Lei and N.A. Obers, Nonrelativistic Chern-Simons theories and three-dimensional Horava-Lifshitz gravity, Phys. Rev. D 94 (2016) 065027 [arXiv: 1604.08054] [INSPIRE].

[37] E.A. Bergshoeff and J. Rosseel, Three-Dimensional Extended Bargmann Supergravity, Phys. Rev. Lett. 116 (2016) 251601 [arXiv: 1604.08042] [INSPIRE].

[38] J. Hartong, Y. Lei, N.A. Obers and G. Oling, Zooming in on $A d S_{3} / C F T_{2}$ near a BPS bound, JHEP 05 (2018) 016 [arXiv:1712.05794] [INSPIRE].

[39] J. Matulich, S. Prohazka and J. Salzer, Limits of three-dimensional gravity and metric kinematical Lie algebras in any dimension, JHEP 07 (2019) 118 [arXiv:1903.09165] [INSPIRE].

[40] E. Bergshoeff, D. Grumiller, S. Prohazka and J. Rosseel, Three-dimensional Spin-3 Theories Based on General Kinematical Algebras, JHEP 01 (2017) 114 [arXiv:1612.02277] [INSPIRE].

[41] J. Gomis, D. Hidalgo and P. Salgado-ReboLledó, Non-relativistic and Carrollian limits of Jackiw-Teitelboim gravity, arXiv:2011.15053 [INSPIRE].

[42] K. Isler and C.A. Trugenberger, A Gauge Theory of Two-dimensional Quantum Gravity, Phys. Rev. Lett. 63 (1989) 834 [INSPIRE].

[43] A.H. Chamseddine and D. Wyler, Gauge Theory of Topological Gravity in (1+1)-Dimensions, Phys. Lett. B 228 (1989) 75 [INSPIRE].

[44] J. Salzer, Asymptotic dynamics of two-dimensional dilaton gravity, Ph.D. Thesis, Vienna University of Technology, Vienna Austria (2018), https://inspirehep.net/files/abdb401a4bd06263c705f7772d5afba5.

[45] D. Birmingham, M. Blau, M. Rakowski and G. Thompson, Topological field theory, Phys. Rept. 209 (1991) 129 [INSPIRE]. 
[46] A. Medina and P. Revoy, Algèbres de lie et produit scalaire invariant, Ann. Sci. Éc. Norm. Supér. 18 (1985) 553, http://eudml.org/doc/82165.

[47] J.M. Figueroa-O'Farrill and S. Stanciu, On the structure of symmetric selfdual Lie algebras, J. Math. Phys. 37 (1996) 4121 [hep-th/9506152] [INSPIRE].

[48] H. Bacry and J. Levy-Leblond, Possible kinematics, J. Math. Phys. 9 (1968) 1605 [INSPIRE].

[49] J. Figueroa-O'Farrill and S. Prohazka, Spatially isotropic homogeneous spacetimes, JHEP 01 (2019) 229 [arXiv: 1809.01224] [INSPIRE].

[50] J. Hartong and N.A. Obers, Hořava-Lifshitz gravity from dynamical Newton-Cartan geometry, JHEP 07 (2015) 155 [arXiv:1504.07461] [INSPIRE].

[51] X. Bekaert and K. Morand, Connections and dynamical trajectories in generalised Newton-Cartan gravity II. An ambient perspective, J. Math. Phys. 59 (2018) 072503 [arXiv: 1505. 03739] [INSPIRE].

[52] J. Hartong, Gauging the Carroll Algebra and Ultra-Relativistic Gravity, JHEP 08 (2015) 069 [arXiv: 1505.05011] [INSPIRE].

[53] J. Hartong, Holographic Reconstruction of 3D Flat Space-Time, JHEP 10 (2016) 104 [arXiv: 1511.01387] [INSPIRE].

[54] K. Jensen, Locality and anomalies in warped conformal field theory, JHEP 12 (2017) 111 [arXiv:1710.11626] [INSPIRE].

[55] J. Figueroa-O'Farrill, On the intrinsic torsion of spacetime structures, arXiv:2009.01948 [INSPIRE].

[56] E. Bergshoeff, J. Gomis, B. Rollier, J. Rosseel and T. ter Veldhuis, Carroll versus Galilei Gravity, JHEP 03 (2017) 165 [arXiv:1701.06156] [INSPIRE].

[57] R. Andringa, E. Bergshoeff, S. Panda and M. de Roo, Newtonian Gravity and the Bargmann Algebra, Class. Quant. Grav. 28 (2011) 105011 [arXiv:1011.1145] [INSPIRE].

[58] D. Grumiller and R. Meyer, Ramifications of lineland, Turk. J. Phys. 30 (2006) 349 [hep-th/0604049] [INSPIRE].

[59] D. Cangemi and R. Jackiw, Gauge invariant formulations of lineal gravity, Phys. Rev. Lett. 69 (1992) 233 [hep-th/9203056] [INSPIRE].

[60] H.L. Verlinde, Black holes and strings in two-dimensions, in 6th Marcel Grossmann Meeting on General Relativity (MG6), Kyoto Japan (1991), pg. 178.

[61] R. Jackiw, Gauge theories for gravity on a line, Theor. Math. Phys. 92 (1992) 979 [hep-th/9206093] [INSPIRE].

[62] R. Schrader, The Maxwell group and the quantum theory of particles in classical homogeneous electromagnetic fields, Fortsch. Phys. 20 (1972) 701 [InSPIRE].

[63] H. Bacry, P. Combe and J.L. Richard, Group-theoretical analysis of elementary particles in an external electromagnetic field. 1 . The relativistic particle in a constant and uniform field, Nuovo Cim. A 67 (1970) 267 [INSPIRE].

[64] L. Bianchi, Sugli spazi a tre dimensioni che ammettono un gruppo continuo di movimenti (in Italian), Mem. Mat. Fis. Soc. Ital. Sci. Ser. III Tomo XI (1898) 267. 
[65] L. Bianchi, On the three-dimensional spaces which admit a continuous group of motions, Gen. Rel. Grav. 33 (2001) 2171.

[66] E. Witten, Quantum Field Theory and the Jones Polynomial, Commun. Math. Phys. 121 (1989) 351 [INSPIRE].

[67] S. Elitzur, G.W. Moore, A. Schwimmer and N. Seiberg, Remarks on the Canonical Quantization of the Chern-Simons-Witten Theory, Nucl. Phys. B 326 (1989) 108 [InSPIRE].

[68] H.A. González, D. Grumiller and J. Salzer, Towards a bulk description of higher spin SYK, JHEP 05 (2018) 083 [arXiv:1802.01562] [INSPIRE].

[69] D. Kapec, R. Mahajan and D. Stanford, Matrix ensembles with global symmetries and 't Hooft anomalies from $2 d$ gauge theory, JHEP 04 (2020) 186 [arXiv:1912.12285] [INSPIRE].

[70] J. Cotler and K. Jensen, A theory of reparameterizations for AdS 3 gravity, JHEP 02 (2019) 079 [arXiv: 1808.03263] [INSPIRE].

[71] W. Merbis and M. Riegler, Geometric actions and flat space holography, JHEP 02 (2020) 125 [arXiv: 1912.08207] [INSPIRE].

[72] J.D. Brown and M. Henneaux, Central Charges in the Canonical Realization of Asymptotic Symmetries: An Example from Three-Dimensional Gravity, Commun. Math. Phys. 104 (1986) 207 [INSPIRE].

[73] G. Compère, W. Song and A. Strominger, New Boundary Conditions for AdS3, JHEP 05 (2013) 152 [arXiv: 1303.2662] [INSPIRE].

[74] C. Troessaert, Enhanced asymptotic symmetry algebra of $A d S_{3}$, JHEP 08 (2013) 044 [arXiv:1303.3296] [INSPIRE].

[75] D. Grumiller and M. Riegler, Most general AdS 3 boundary conditions, JHEP 10 (2016) 023 [arXiv:1608.01308] [INSPIRE].

[76] O. Coussaert, M. Henneaux and P. van Driel, The Asymptotic dynamics of three-dimensional Einstein gravity with a negative cosmological constant, Class. Quant. Grav. 12 (1995) 2961 [gr-qc/9506019] [InSPIRE].

[77] G. Barnich and G. Compere, Classical central extension for asymptotic symmetries at null infinity in three spacetime dimensions, Class. Quant. Grav. 24 (2007) F15 [gr-qc/0610130] [INSPIRE].

[78] L. Feher, L. O'Raifeartaigh, P. Ruelle, I. Tsutsui and A. Wipf, On the general structure of Hamiltonian reductions of the WZNW theory, hep-th/9112068 [INSPIRE].

[79] M. Henneaux and C. Teitelboim, Quantization of gauge systems, Princeton University Press, Princeton U.S.S. (1992).

[80] D. Grumiller, R. McNees, J. Salzer, C. Valcárcel and D. Vassilevich, Menagerie of Ad $S_{2}$ boundary conditions, JHEP 10 (2017) 203 [arXiv: 1708.08471] [INSPIRE].

[81] J. Cotler, K. Jensen and A. Maloney, Low-dimensional de Sitter quantum gravity, JHEP 06 (2020) 048 [arXiv: 1905.03780] [INSPIRE].

[82] S. Carlip, The dynamics of supertranslations and superrotations in $2+1$ dimensions, Class. Quant. Grav. 35 (2018) 014001 [arXiv: 1608.05088] [INSPIRE].

[83] K. Nguyen and J. Salzer, The Effective Action of Superrotation Modes, arXiv:2008.03321 [INSPIRE]. 
[84] H. Afshar, S. Detournay, D. Grumiller and B. Oblak, Near-Horizon Geometry and Warped Conformal Symmetry, JHEP 03 (2016) 187 [arXiv:1512.08233] [INSPIRE].

[85] H.R. Afshar, Warped Schwarzian theory, JHEP 02 (2020) 126 [arXiv:1908.08089] [INSPIRE].

[86] H. Afshar, H.A. González, D. Grumiller and D. Vassilevich, Flat space holography and the complex Sachdev-Ye-Kitaev model, Phys. Rev. D 101 (2020) 086024 [arXiv:1911.05739] [INSPIRE].

[87] C. Duval, G.W. Gibbons, P.A. Horvathy and P.M. Zhang, Carroll versus Newton and Galilei: two dual non-Einsteinian concepts of time, Class. Quant. Grav. 31 (2014) 085016 [arXiv: 1402.0657] [INSPIRE].

[88] A. Bagchi, R. Basu, A. Mehra and P. Nandi, Field Theories on Null Manifolds, JHEP 02 (2020) 141 [arXiv: 1912.09388] [INSPIRE].

[89] N. Ozdemir, M. Ozkan, O. Tunca and U. Zorba, Three-Dimensional Extended Newtonian (Super)Gravity, JHEP 05 (2019) 130 [arXiv: 1903.09377] [INSPIRE].

[90] L. Ravera, AdS Carroll Chern-Simons supergravity in $2+1$ dimensions and its flat limit, Phys. Lett. B 795 (2019) 331 [arXiv:1905.00766] [INSPIRE].

[91] P. Concha and E. Rodríguez, Non-Relativistic Gravity Theory based on an Enlargement of the Extended Bargmann Algebra, JHEP 07 (2019) 085 [arXiv: 1906.00086] [InSPIRE].

[92] J. Gomis, A. Kleinschmidt, J. Palmkvist and P. Salgado-ReboLledó, Newton-Hooke/Carrollian expansions of (A)dS and Chern-Simons gravity, JHEP 02 (2020) 009 [arXiv: 1912.07564] [INSPIRE].

[93] N. Mohammedi, On bosonic and supersymmetric current algebras for nonsemisimple groups, Phys. Lett. B 325 (1994) 371 [hep-th/9312182] [INSPIRE].

[94] J.M. Figueroa-O'Farrill and S. Stanciu, Nonsemisimple Sugawara constructions, Phys. Lett. $B 327$ (1994) 40 [hep-th/9402035] [INSPIRE].

[95] P. Chaturvedi, I. Papadimitriou, W. Song and B. Yu, AdS $S_{3}$ gravity and the complex $S Y K$ models, arXiv:2011.10001 [INSPIRE].

[96] D. Grumiller, W. Merbis and M. Riegler, Most general flat space boundary conditions in three-dimensional Einstein gravity, Class. Quant. Grav. 34 (2017) 184001 [arXiv: 1704.07419] [INSPIRE].

[97] A. Strominger, Lectures on the Infrared Structure of Gravity and Gauge Theory, arXiv: 1703.05448 [INSPIRE].

[98] M. Guica, An integrable Lorentz-breaking deformation of two-dimensional CFTs, SciPost Phys. 5 (2018) 048 [arXiv:1710.08415] [INSPIRE].

[99] D.J. Gross, J. Kruthoff, A. Rolph and E. Shaghoulian, $T \bar{T}$ in $A d S_{2}$ and Quantum Mechanics, Phys. Rev. D 101 (2020) 026011 [arXiv:1907.04873] [InSPIRE].

[100] D.J. Gross, J. Kruthoff, A. Rolph and E. Shaghoulian, Hamiltonian deformations in quantum mechanics, $T \bar{T}$, and the SYK model, Phys. Rev. D 102 (2020) 046019 [arXiv: 1912.06132] [INSPIRE].

[101] D. Grumiller and R. McNees, Universal flow equations and chaos bound saturation in $2 d$ dilaton gravity, JHEP 01 (2021) 112 [arXiv:2007.03673] [INSPIRE]. 
[102] M. Cárdenas, O. Fuentealba, H.A. González, D. Grumiller, C. Valcárcel and D. Vassilevich, Boundary theories for dilaton supergravity in 2D, JHEP 11 (2018) 077 [arXiv:1809.07208] [INSPIRE].

[103] K. Alkalaev and X. Bekaert, On BF-type higher-spin actions in two dimensions, JHEP 05 (2020) 158 [arXiv: 2002.02387] [INSPIRE].

[104] G. Barnich and M. Henneaux, Consistent couplings between fields with a gauge freedom and deformations of the master equation, Phys. Lett. B 311 (1993) 123 [hep-th/9304057] [INSPIRE].

[105] N. Ikeda, Two-dimensional gravity and nonlinear gauge theory, Annals Phys. 235 (1994) 435 [hep-th/9312059] [INSPIRE].

[106] P. Schaller and T. Strobl, Poisson structure induced (topological) field theories, Mod. Phys. Lett. A 9 (1994) 3129 [hep-th/9405110] [INSPIRE].

[107] G.P. Ovando, Lie algebras with ad-invariant metrics. A survey, arXiv:1512.03997.

[108] A. Medina, Groupes de Lie munis de métriques bi-invariantes, Tohoku Math. J. (2) $\mathbf{3 7}$ (1985) 405.

[109] J.M. Figueroa-O'Farrill, On parallelizable NS-NS backgrounds, Class. Quant. Grav. 20 (2003) 3327 [hep-th/0305079] [INSPIRE].

[110] I. Kath and M. Olbrich, Metric Lie algebras with maximal isotropic centre, Math. Z. 246 (2004) 23.

[111] I. Kath and M. Olbrich, Metric Lie algebras and quadratic extensions, Transform. Groups 11 (2006) 87.

[112] D. Hansen, J. Hartong and N.A. Obers, Action Principle for Newtonian Gravity, Phys. Rev. Lett. 122 (2019) 061106 [arXiv: 1807.04765] [INSPIRE].

[113] J.A. de Azcarraga, J.M. Izquierdo, M. Picón and O. Varela, Generating Lie and gauge free differential (super)algebras by expanding Maurer-Cartan forms and Chern-Simons supergravity, Nucl. Phys. B 662 (2003) 185 [hep-th/0212347] [INSPIRE].

[114] F. Izaurieta, E. Rodriguez and P. Salgado, Expanding Lie (super)algebras through Abelian semigroups, J. Math. Phys. 47 (2006) 123512 [hep-th/0606215] [INSPIRE].

[115] O. Khasanov and S. Kuperstein, (In)finite extensions of algebras from their Inonu-Wigner contractions, J. Phys. A 44 (2011) 475202 [arXiv:1103.3447] [InSPIRE].

[116] S. Prohazka, Chern-Simons Holography, Ph.D. Thesis, Vienna University of Technology, Vienna Austria (2017) [arXiv: 1710.11110] [INSPIRE].

[117] A. Barducci, R. Casalbuoni and J. Gomis, Nonrelativistic k-contractions of the coadjoint Poincaré algebra, Int. J. Mod. Phys. A 35 (2020) 2050009 [arXiv:1910.11682] [INSPIRE].

[118] E. Bergshoeff, J. Gomis and P. Salgado-ReboLledó, Non-relativistic limits and three-dimensional coadjoint Poincaré gravity, Proc. Roy. Soc. Lond. A 476 (2020) 20200106 [arXiv: 2001.11790] [inSPIRE].

[119] A. Barducci, R. Casalbuoni and J. Gomis, A particle model with extra dimensions from Coadjoint Poincaré Symmetry, JHEP 08 (2020) 092 [arXiv:2006.11725] [INSPIRE]. 